\title{
Behavioral perfect equilibrium in Bayesian games
}

Citation for published version (APA):

Bajoori, E., Flesch, J., \& Vermeulen, D. (2016). Behavioral perfect equilibrium in Bayesian games. Games and Economic Behavior, 98, 78-109. https://doi.org/10.1016/j.geb.2016.06.002

Document status and date:

Published: 01/07/2016

DOI:

10.1016/j.geb.2016.06.002

Document Version:

Publisher's PDF, also known as Version of record

Document license:

Taverne

\section{Please check the document version of this publication:}

- A submitted manuscript is the version of the article upon submission and before peer-review. There can be important differences between the submitted version and the official published version of record.

People interested in the research are advised to contact the author for the final version of the publication, or visit the DOI to the publisher's website.

- The final author version and the galley proof are versions of the publication after peer review.

- The final published version features the final layout of the paper including the volume, issue and page numbers.

Link to publication

\footnotetext{
General rights rights.

- You may freely distribute the URL identifying the publication in the public portal. please follow below link for the End User Agreement:

www.umlib.nl/taverne-license

Take down policy

If you believe that this document breaches copyright please contact us at:

repository@maastrichtuniversity.nl

providing details and we will investigate your claim.
}

Copyright and moral rights for the publications made accessible in the public portal are retained by the authors and/or other copyright owners and it is a condition of accessing publications that users recognise and abide by the legal requirements associated with these

- Users may download and print one copy of any publication from the public portal for the purpose of private study or research.

- You may not further distribute the material or use it for any profit-making activity or commercial gain

If the publication is distributed under the terms of Article $25 \mathrm{fa}$ of the Dutch Copyright Act, indicated by the "Taverne" license above, 


\title{
Behavioral perfect equilibrium in Bayesian games
}

\author{
Elnaz Bajoori ${ }^{\mathrm{a}}$, János Flesch ${ }^{\mathrm{b}}$, Dries Vermeulen ${ }^{\mathrm{b}, *}$ \\ a University of Bath, Dept. of Economics, Bath, UK \\ b Maastricht University, School of Business and Economics, Dept. of Quantitative Economics, P.O. Box 616, 6200 MD Maastricht, \\ The Netherlands
}

\section{A R T I C L E I N F O}

Article history:

Received 15 November 2013

Available online 11 June 2016

\section{JEL classification:}

$\mathrm{C} 72$

\section{Keywords:}

Trembling hand perfect equilibrium Bayesian game with infinite type spaces Behavior strategy

Second-price auction with incomplete information

\begin{abstract}
A B S T R A C T
We develop the notion of perfect Bayesian Nash Equilibrium-perfect BNE-in general Bayesian games. We test perfect BNE against the criteria laid out by Kohlberg and Mertens (1986). We show that, for a focal class of Bayesian games, perfect BNE exists. Moreover, when payoffs are continuous, perfect BNE is limit undominated for almost every type.

We illustrate the use of perfect BNE in the context of a second-price auction with interdependent values. Perfect BNE selects the unique pure strategy equilibrium in continuous strategies that separates types. Moreover, when valuations become independent, the equilibrium converges to the classical truthful dominant strategy equilibrium.

We also show that less intuitive equilibria in which types are pooled are ruled out by our selection criterion. We further argue that standard selection criteria for second-price auctions have no bite here. Bidders have no dominant strategies, and the separating equilibrium is not sincere.
\end{abstract}

(c) 2016 Elsevier Inc. All rights reserved.

\section{Introduction}

For normal form games with finite action spaces, Nash (1951) introduced the concept of Nash equilibrium and proved its existence. Since Nash equilibria are not always intuitive as a solution, several refinements of Nash equilibrium have been proposed in the literature. Among these, perfect equilibrium (Selten, 1975) is one of the most commonly used refinement concepts.

Many applications of Nash equilibrium and its refinements are in the context of incomplete information games. Examples of games with incomplete information are signaling games (Kreps and Wilson, 1982), principal-agent models (Laffont and Martimort, 2001), and models of reputation such as the chain store paradox (Selten, 1978; Govindan, 1995). In particular in auction design refinements of Nash equilibrium are used to reduce the number of equilibria and rule out the less intuitive or desirable equilibria. For example in the Vickrey auction, selection on dominant strategies singles out the truthful equilibrium, and rules out the (many) ex post equilibria in which partial pooling of types occurs. Also Jackson et al. (2002) employ perfect equilibrium in one of their examples to eliminate equilibria in which players may bid above their maximum possible valuation.

Our aim is to develop the refinement of perfect equilibrium in the context of such applications, in particular auction design. We focus on the game theoretic framework of Bayesian games, the standard tool to model incomplete information. We study the class of Bayesian games in which players, after the information phase in which they learn their type, play

\footnotetext{
* Corresponding author.

E-mail addresses: e.bajoori@bath.ac.uk (E. Bajoori), j.flesch@maastrichtuniversity.nl (J. Flesch), d.vermeulen@maastrichtuniversity.nl (D. Vermeulen).
} 
a one-shot game. We develop the notion of perfect equilibrium for such games, and in effect we propose three possible variations. We study the relations between these variations, and illustrate their use in the context of a second price auction.

When applying refinements to auctions and incomplete information games there are typically two problems to tackle. First, such games often feature discontinuities in the payoff functions. This issue is addressed in for example Reny (1999) and Jackson et al. (2002).

Second, incomplete information games, especially auctions, often have continuum type spaces and action spaces. Refinements for incomplete information games, such as for example sequential equilibrium (Kreps and Wilson, 1982) and perfect Bayesian equilbrium (Fudenberg and Tirole, 1991), are as a rule developed for finite games, and as such not immediately applicable in the context of auction theory and similar economic applications with incomplete information. An influential first attempt to generalize known equilibrium refinements to more general classes of games is the working paper by Myerson and Reny (2011). Our paper also contributes to the development of equilibrium refinement for incomplete information games, and generalizes the notion of perfect equilibrium to the class of Bayesian games.

We take a conceptual view. A central solution concept in Bayesian games is that of Bayesian Nash Equilibrium (BNE), a direct extension of Nash equilibrium to games with incomplete information. A BNE is a profile of behavior strategies, one for each player, such that each player's strategy, given any type for this player, is an expected value maximizer given the strategies of his opponents, where expectations are taken over all possible types of the opponents. ${ }^{2}$ So, the best response property that characterizes the equilibrium concept is required at the interim stage when the player already knows his own type. $^{3}$

In this paper we work with interim probabilities, unless mentioned otherwise. Our goal is to define the notion of perfect BNE in Bayesian games, analyze its properties, and illustrate its predictive power in an elaborate example of a second price auction with interdependent values. Our work is motivated by the observation that, under incomplete information, standard selection criteria in auction design, such as dominant strategy equilibrium and truthful reporting, no longer have a bite. Also notions such as sequential equilibrium and perfect Bayesian equilibrium (Kreps and Wilson, 1982; Fudenberg and Tirole, 1991; Bonanno, 2013) only have selective power in the context of extensive form games with multiple rounds. Thus in the setting of single round sealed bid auctions these refinements do not reduce the set of equilibria. According to the theory of refinements, the selection criterion that best suits our context is perfect equilibrium. This is the solution concept we develop for the class of Bayesian games.

We base our approach on completely mixed behavior strategies, i.e. behavior strategies which prescribe a completely mixed probability measure for each possible type. ${ }^{4}$ We first define perfection for general behavior strategy profiles, so not necessarily for BNEs yet. Roughly speaking, a behavior strategy profile $\beta$ is called perfect if there is a sequence $\left(\beta^{k}\right)_{k=1}^{\infty}$ of completely mixed behavior strategy profiles such that, for every player $i$, the distance between $\beta_{i}^{k}$ and $\beta_{i}$ and the distance between $\beta_{i}^{k}$ and player $i$ 's best responses against $\beta^{k}$ both converge to 0 . We measure distance on the set of actions by means of the weak metric, and we consider almost everywhere convergence on the set of types. In general, such a profile $\beta$ is not necessarily a BNE, since we did not impose strong conditions on the type and actions spaces and on the payoff functions in the Bayesian game. If $\beta$ is also a BNE, then we call $\beta$ a perfect BNE.

In the first part of the paper we define perfect BNE in Bayesian games, and test our concept against the list of requirements for equilibrium refinements proposed by Kohlberg and Mertens (1986). We prove that perfect BNE exists for Bayesian games with finite action spaces, at most countable type spaces, and continuous payoffs. We also prove that a perfect BNE is limit undominated for almost every type, provided that the payoff functions are continuous. We further examine the two alternative notions of perfection, and their relations to perfect BNE, where convergence on the set of types is required to hold either pointwisely for each type, or uniformly for all types. In addition, we briefly discuss finite Bayesian games, i.e. when there are only finitely many types and actions.

In the second part of the paper, we illustrate the use of perfection as a tool to select the more intuitive BNEs in Bayesian games. We do so by examining a sealed-bid second-price auction with two bidders. In this auction, the valuation function of each bidder does not only depend on his own type, but also on the type of his opponent. More precisely, for each $i=1,2$, bidder $i$ 's valuation is given by $v_{i}=5+t_{i}-\alpha t_{j}$, where $\alpha \in(0,1)$ and $j \neq i$. Jackson et al. $(2002,2004)$ considered the first price version of this auction with $\alpha=4$ and proved that no BNE exists if each player has a positive probability to win in case of a tie. However, they show that there exists a simple BNE in this auction when the tie breaking rule depends on types.

This second-price auction admits multiple BNEs, but we find that perfection selects a BNE $\beta$ that is unique in a certain class of separating strategy profiles. We also show that perfection rules out many BNEs in which pooling occurs among

\footnotetext{
1 The result in Myerson and Reny (2011) covers a large class of incomplete information games. Their solution concept assigns finitely additive probability measures, while our construction remains within the environment of countably additive probability measures.

2 Behavior strategies are much in the spirit of Bayesian games. A behavior strategy of a player prescribes a probability measure on his set of actions, depending on the type of this player, that satisfies an additional measurability assumption. An alternative approach based on distributional strategies is investigated in Bajoori (2011).

3 A similar but weaker concept arises if one calculates ex-ante probabilities, and only requires the best response property for each player before he receives his own type. This approach is investigated for example by Reny (2011) and by Milgrom and Weber (1985).

4 Thus, each player takes into account mistakes by all possible types of his opponents on their action choices. This approach is more selective than considering such mistakes for only certain types.
} 
types. We highlight the subtleties involved in the choice of sequence of completely mixed behavior strategy profiles $\left(\beta^{k}\right)_{k=1}^{\infty}$, because the most straightforward candidate for this sequence, the uniform distribution, does not select a BNE.

We also show that standard complete information selection criteria such as dominant strategies and truthful reporting do not apply here. Sincere reporting is not an equilibrium, the selected BNE $\beta$ is not sincere, and neither player has a dominant strategy in this auction. This emphasizes the necessity to employ more sophisticated refinement techniques such as perfect BNE to select among many BNEs in this auction.

As a final remark, we already observed that for example Reny (2011) and Milgrom and Weber (1985) take an ex ante approach. However, both from a conceptual and a computational view, the interim approach seems to be preferable. Conceptual, since perfect BNE is defined directly at the level of behavior strategies. Computational, since perfect BNE is calculated at the level of the Bayesian game itself, not in the context of the induced strategic form game. Our computations in the second price auction emphasize these observations.

RELATED LITERATURE Balder (1988) generalizes Milgrom and Weber's results and proves the existence of BNE in behavior strategies by making use of the theory of weak convergence of transition probabilities. The same theory has been used in Balder (2002) to obtain existence results for Cournot-Nash equilibria.

Simon and Stinchcombe (1995) define perfect equilibrium for strategic form games with compact action spaces. They discuss two essentially different approaches. The first approach is a direct generalization of Selten's original definition, based on the notion of completely mixed strategies. The second approach to perfect equilibrium, by Simon and Stinchcombe referred to as the finitistic approach, uses the notion of an $\varepsilon$-perfect equilibrium in finite approximations of the original game. They show existence of these notions of perfect equilibrium, and investigate the properties of and relations between the various resulting solution concepts.

Bajoori et al. (2013) examine the two approaches proposed in Simon and Stinchcombe (1995) in further detail and provide an improved definition of the finitistic approach. Their results seem to imply a critique on the finitistic approach.

Jackson et al. (2002) study games with incomplete information and discontinuous payoffs. In the incomplete information setting discontinuities often arise from indifferences between players' choices and the particular resolution of such indifferences in the description of the game. The paper shows in several examples that the resulting discontinuities may cause extreme behavior in equilibrium, or even non-existence of equilibrium. They show that the introduction of a communication phase before the start of the game may mitigate strategic effects, and restore existence of equilibrium with truthful reporting.

Reny (2011) shows, under general conditions, the existence of a monotone pure-strategy equilibrium. The main innovations in the paper are that the result is shown to hold for a wide class of partially ordered spaces, and that best response sets only need to be join-closed (meaning that the join of two best responses is again a best response).

Myerson and Reny (2011) develop the concept of sequential equilibrium for a very general class of multistage games with incomplete information. They prove existence of sequential equilibrium in terms of induced finitely additive conditional probability distributions.

Fudenberg and Tirole (1991) define perfect Bayesian equilibrium and sequential equilibrium for finite Bayesian multiperiod games, and show that these notions coincide when each player only has two types.

Our paper is structured as follows. First, we discuss some preliminary notions in Section 2 and present the model of Bayesian games in Section 3. Then, in Section 4, we define the concept of perfect BNE and analyze its properties. In Section 5, we apply our results to the above mentioned auction. The paper ends with an extensive appendix, which contains the proofs of several technical results that we use in earlier parts of the paper.

NOTATION For a metric space $(X, d)$, the set of probability measures on the Borel $\sigma$-field $\Sigma$ on $X$ is denoted by $\Delta(X)$. A probability measure $\mu \in \Delta(X)$ is completely mixed if $\mu(U)>0$ for every nonempty open subset $U$ of $X$. The weak (Prokhorov) metric $\rho^{w}$ on $\Delta(X)$ is defined for every $\mu, v \in \Delta(X)$ by

$$
\rho^{w}(\mu, \nu)=\inf \left\{\varepsilon>0 \mid \forall B \in \Sigma: \mu(B) \leq \nu\left(B^{\varepsilon}\right)+\varepsilon \text { and } \nu(B) \leq \mu\left(B^{\varepsilon}\right)+\varepsilon\right\} .
$$

Let $\mu_{n} \in \Delta(X)$ for every $n \in \mathbb{N}$ and let $\mu \in \Delta(X)$. It is known that if the sequence $\mu_{n}$ converges to $\mu$ with respect to $\rho^{w}$, then $\int_{X} f(x) \mu_{n}(d x)$ converges to $\int_{X} f(x) \mu(d x)$ for every bounded and continuous function $f: X \rightarrow \mathbb{R}$. Conversely, if $X$ is separable and $\int_{X} f(x) \mu_{n}(d x)$ converges to $\int_{X} f(x) \mu(d x)$ for every bounded and Lipschitz function $f: X \rightarrow \mathbb{R}$, then $\mu_{n}$ converges to $\mu$ with respect to $\rho^{w}$. Further, if $X$ is compact, then so is $\Delta(X)$ with respect to $\rho^{w}$ (cf. Prokhorov, 1956 ; Parthasarathy, 1967).

\section{Bayesian games}

Definition 1. A Bayesian game is a tuple $\Gamma=\left(N,\left(T_{i}, d_{T_{i}}\right)_{i \in N},\left(A_{i}, d_{A_{i}}\right)_{i \in N},\left(\mu_{i}\right)_{i \in N},\left(\Pi_{i}\right)_{i \in N}\right)$ where:

1. $N=\{1,2, . ., n\}$ is the set of players.

2. $T_{i}$ is a nonempty set of player $i$ 's possible types with metric $d_{T_{i}}$. Let $\mathcal{T}_{i}$ denote the induced Borel $\sigma$-field on $T_{i}$, $T=\times_{i=1}^{n} T_{i}$, and $\mathcal{T}=\otimes_{i=1}^{n} \mathcal{T}_{i}$.

3. $A_{i}$ is a nonempty set of player $i$ 's actions with metric $d_{A_{i}}$. Let $\mathcal{A}_{i}$ denote the induced Borel $\sigma$-field on $A_{i}$, $A=\times_{i=1}^{n} A_{i}$, and $\mathcal{A}=\otimes_{i=1}^{n} \mathcal{A}_{i}$. 
4. $\mu_{i}$ is a probability measure on $\left(T_{i}, \mathcal{T}_{i}\right)$ for player $i$. Let $\mu=\times_{i=1}^{n} \mu_{i}$ be the product measure on $(T, \mathcal{T})$.

5. $\Pi_{i}: T \times A \rightarrow \mathbb{R}$ is player $i$ 's payoff function, bounded and measurable with respect to $\mathcal{T} \otimes \mathcal{A}$. Let $\Pi=\left(\Pi_{i}\right)_{i=1}^{n}$.

The Bayesian game $\Gamma$ is played as follows: First, nature draws a type $t_{i} \in T_{i}$ for each player $i$ according to the probability measure $\mu_{i}$. Each player $i$ learns his own type $t_{i}$, but not the types of the other players. Then, each player $i$ chooses an action $a_{i} \in A_{i}$, simultaneously and independently. Finally, depending on the types $t=\left(t_{1}, \ldots, t_{n}\right)$ and the chosen actions $a=\left(a_{1}, \ldots, a_{n}\right)$, each player $i$ receives the payoff $\Pi_{i}(t, a)$.

Now, we discuss different classes of strategies for the players. We start with the simplest ones.

Definition 2. A pure strategy for player $i$ is a measurable function $p_{i}: T_{i} \rightarrow A_{i}$.

Thus, a pure strategy prescribes one specific action depending on the player's type. Now we move on to the strategies which use some randomization for the choice of an action. In the finite version of the model, i.e., when $T_{i}$ and $A_{i}$ are finite, a mixed strategy is a probability measure on the set of pure strategies, whereas a behavior strategy prescribes, for each possible type, a probability measure on the set of available actions. Aumann (1964) observed however that, for our infinite model, the above view of a mixed strategy leads to measure theoretic problems and does not provide an acceptable definition. Instead, a mixed strategy should be modeled by a random variable with values in the set of pure strategies, whose domain is a probability measure space that is used as the randomization device. This is the underlying idea of the definition of a mixed strategy for player $i$ as a measurable function $\alpha_{i}: T_{i} \times[0,1] \rightarrow A_{i}$, where the uniform distribution is imposed on $[0,1]$. The interpretation of a mixed strategy $\alpha_{i}$ is that, after observing his own type $t_{i}$ and drawing a randomization-variable $s_{i}$ from $[0,1]$ according to uniform distribution, player $i$ plays $\alpha_{i}\left(t_{i}, s_{i}\right)$. Note that, for every $s_{i} \in[0,1]$, the section function $\alpha_{i}\left(\cdot, s_{i}\right): T_{i} \rightarrow A_{i}$ is a pure strategy. Behavior strategies are defined with similar considerations:

Definition 3. A behavior strategy for player $i$ is a function $\beta_{i}: T_{i} \times \mathcal{A}_{i} \rightarrow[0,1]$ such that

1. the section function $\beta_{i}\left(t_{i}, \cdot\right): \mathcal{A}_{i} \rightarrow[0,1]$ is a probability measure for every $t_{i} \in T_{i}$,

2. the section function $\beta_{i}(\cdot, B): T_{i} \rightarrow[0,1]$ is measurable for every $B \in \mathcal{A}_{i}{ }^{5}$

A behavior strategy $\beta_{i}$ prescribes, depending on player $i$ 's type $t_{i}$, to choose an action according to the probability measure $\beta_{i}\left(t_{i}, \cdot\right)$. The second condition in the definition is included so that the ex-ante probability that player $i$ 's action falls into a set $B \in \mathcal{A}_{i}$ exists and is equal to $\int_{T_{i}} \beta_{i}\left(t_{i}, B\right) \mu_{i}\left(d t_{i}\right)$. We will usually define a behavior strategy by specifying the section function $\beta_{i}\left(t_{i}, \cdot\right)$ for every type $t_{i} \in T_{i}$. Behavior strategies are well suited for our purpose to define perfect equilibrium. Indeed, the probability measures $\beta_{i}\left(t_{i}, \cdot\right)$ are sufficient to describe player $i$ 's behavior. Moreover, as Aumann (1964) showed, there is a many-to-one mapping from mixed to behavior strategies that preserves the players' expected payoffs, so mixed strategies would have no significant added value. For these reasons, we build our definitions on behavior strategies. From now on, by a strategy we will always mean a behavior strategy, unless mentioned otherwise.

Definition 4. A strategy $\beta_{i}$ for player $i$ is called deterministic if, for every type $t_{i} \in T_{i}$, there is an action $a_{i, t_{i}} \in A_{i}$ such that $\beta_{i}\left(t_{i}, \cdot\right)$ is the Dirac measure on $a_{i, t_{i}}$. A strategy $\beta_{i}$ is called completely mixed if the section function $\beta_{i}\left(t_{i}, \cdot\right): \mathcal{A}_{i} \rightarrow[0,1]$ is a completely mixed probability measure for every $t_{i} \in T_{i}$. The vector $\beta=\left(\beta_{1}, \beta_{2}, \ldots, \beta_{n}\right)$, where $\beta_{i}$ is a strategy of player $i$, is called a strategy profile.

Each pure strategy $p_{i}$ naturally induces a deterministic strategy $\beta_{i}$, for which $\beta_{i}\left(t_{i}, \cdot\right)$ is the Dirac measure on $p_{i}\left(t_{i}\right)$ for every type $t_{i} \in T_{i}$.

For every strategy profile $\beta$ and every player $i \in N$, we write $\beta_{-i}=\left(\beta_{j}\right)_{j \in N \backslash\{i\}}$ to denote the profile consisting of strategies of the players in $N \backslash\{i\}$. Further, we use $\mathbb{E}_{\tau_{-i}}\left(\Pi_{i} \mid t_{i}, \beta_{i}\right)$ to denote player $i$ 's expected payoff, given his type $t_{i}$ and his strategy $\beta_{i}$, against a strategy profile $\tau_{-i}$. Thus,

$$
\mathbb{E}_{\tau_{-i}}\left(\Pi_{i} \mid t_{i}, \beta_{i}\right)=\iint_{T_{-i}} \int_{A_{-i}} \int_{A_{i}} \Pi_{i}(t, a) \beta_{i}\left(t_{i}, d a_{i}\right) \tau_{-i}\left(t_{-i}, d a_{-i}\right) \mu_{-i}\left(d t_{-i}\right) .
$$

In the expected payoff above, by integrals with respect to $\tau_{-i}\left(t_{-i}, d a_{-i}\right)$ and $\mu_{-i}\left(d t_{-i}\right)$ we mean the iterated integrals with respect to $\tau_{j}\left(t_{j}, d a_{j}\right)$ and $\mu_{j}\left(d t_{j}\right)$ for all $j \neq i$. Fubini's Theorem and Theorem 10.2.1 in Dudley (2002) guarantee the existence of the iterated integrals in the expression above, and also that the order of integration with respect to $\beta_{i}\left(t_{i}, d a_{i}\right)$

\footnotetext{
${ }^{5}$ As the sets $\left\{t_{i} \mid \beta_{i}\left(t_{i}, E_{i}\right) \geq r\right\}$ are measurable in the type space $T_{i}$ for every measurable subset of actions $E_{i} \subseteq A_{i}$, there is no difference between modeling behavior strategies as measurable functions from $T_{i}$ to $\Delta\left(A_{i}\right)$ or as in Definition 3 in terms of probability kernels.
} 
and $\tau_{j}\left(t_{j}, d a_{j}\right), j \neq i$, is not relevant. In the special case where player $i$ uses a deterministic strategy with corresponding pure strategy $p_{i}$, player $i$ 's expected payoff is denoted simply by $\mathbb{E}_{\tau_{-i}}\left(\Pi_{i} \mid t_{i}, p_{i}\right)$, and it is equal to

$$
\mathbb{E}_{\tau_{-i}}\left(\Pi_{i} \mid t_{i}, p_{i}\right)=\iint_{T_{-i}} \int_{A_{-i}} \Pi_{i}\left(t,\left(p_{i}\left(t_{i}\right), a_{-i}\right)\right) \tau_{-i}\left(t_{-i}, d a_{-i}\right) \mu_{-i}\left(d t_{-i}\right) .
$$

Since, in the expected payoffs $\mathbb{E}_{\tau_{-i}}\left(\Pi_{i} \mid t_{i}, \beta_{i}\right)$ and $\mathbb{E}_{\tau_{-i}}\left(\Pi_{i} \mid t_{i}, p_{i}\right)$, it is irrelevant how player $i$ chooses his actions for types other than $t_{i}$, we can naturally define $\mathbb{E}_{\tau_{-i}}\left(\Pi_{i} \mid t_{i}, \sigma_{i}\right)$ and $\mathbb{E}_{\tau_{-i}}\left(\Pi_{i} \mid t_{i}, a_{i}\right)$ for every probability measure $\sigma_{i}$ on $\left(A_{i}, \mathcal{A}_{i}\right)$ and respectively for every action $a_{i} \in A_{i}$.

A probability measure $\sigma_{i}$ on $\left(A_{i}, \mathcal{A}_{i}\right)$ is called a best response of player $i$ for type $t_{i} \in T_{i}$ against a strategy profile $\tau_{-i}$, if for every probability measure $\sigma_{i}^{\prime}$ on $\left(A_{i}, \mathcal{A}_{i}\right)$ we have

$$
\mathbb{E}_{\tau_{-i}}\left(\Pi_{i} \mid t_{i}, \sigma_{i}\right) \geq \mathbb{E}_{\tau_{-i}}\left(\Pi_{i} \mid t_{i}, \sigma_{i}^{\prime}\right) .
$$

The set of such best responses is denoted by $B R_{i}\left(t_{i}, \tau_{-i}\right)$. For a strategy profile $\tau$, we will also use the notation $B R_{i}\left(t_{i}, \tau\right)$ instead of $B R_{i}\left(t_{i}, \tau_{-i}\right)$.

A strategy $\beta_{i}$ is called a best response of player $i$ against a strategy profile $\tau_{-i}$, if $\beta_{i}\left(t_{i}, \cdot\right) \in B R_{i}\left(t_{i}, \tau_{-i}\right)$ for every $t_{i} \in T_{i}$. The set of such best responses is denoted by $B R_{i}\left(\tau_{-i}\right)$. For a strategy profile $\tau$, we will also use the notation $B R_{i}(\tau)$ instead of $B R_{i}\left(\tau_{-i}\right)$. Note that all these best response sets can be empty, which is illustrated by the following simple example.

Example 5. Consider the following Bayesian game with only one player: $T_{1}=\left\{t_{1}\right\}, A_{1}=[0,1], \Pi_{1}\left(t_{1}, x\right)=x$ for every $x \in[0,1)$, and $\Pi_{1}\left(t_{1}, 1\right)=0$. In this game, the set of the best responses (that is, optimal strategies) of player 1 is empty.

Now we define a central solution concept of Bayesian games, namely the concept of Bayesian Nash equilibrium.

Definition 6. A strategy profile $\beta=\left(\beta_{1}, \beta_{2}, \ldots, \beta_{n}\right)$ is called a Bayesian Nash equilibrium (BNE), if $\beta_{i}$ is a best response of player $i$ against $\beta$, for every player $i$.

Remarks. There are Bayesian games which admit no BNE at all, for instance the game in Example 5. In many Bayesian games of economic interest, however, there exist multiple BNEs, and some of them are arguably more intuitive than others. As mentioned before, our goal is to develop the definition of perfection for BNEs, which can be a useful tool in such games to distinguish the more intuitive BNEs.

We assumed in our model, for simplicity, that the types of the players are drawn independently, and thus the distribution of the type-vectors is described by a product measure $\mu$ on $(T, \mathcal{T})$. Our results can be extended to the case where the distribution of the type-vectors is described by a probability measure $v$ on $(T, \mathcal{T})$ such that $v$ is has a density $f>0$ with respect to the product $\mu$ of the marginal probabilities. This is called diffuse information, and allows for certain kinds of correlation between types of the players. Thus, for every type $t_{i} \in T_{i}$ we have a function $g_{i}\left(t_{i}\right): T_{-i} \rightarrow \mathbb{R}$ defined by

$$
g_{i}\left(t_{i}\right)\left(t_{-i}\right)=\frac{f\left(t_{-i}, t_{i}\right)}{\int_{T_{-i}} f\left(s_{-i}, t_{i}\right) \mu_{-i}\left(d s_{-i}\right)} .
$$

Then for every type $t_{i} \in T_{i}$ and measurable set $S_{-i} \subseteq T_{-i}$,

$$
v\left(S_{-i} \mid t_{i}\right)=\int_{S_{-i}} g_{i}\left(t_{i}\right)\left(t_{-i}\right) \mu_{-i}\left(d t_{-i}\right),
$$

or in other words

$$
v\left(d t \mid t_{i}\right)=g_{i}\left(t_{i}\right)\left(t_{-i}\right) \mu_{-i}\left(d t_{-i}\right) .
$$

Therefore, in deriving the expected payoff $\mathbb{E}_{\tau_{-i}}\left(\Pi_{i} \mid t_{i}, \beta_{i}\right)$, given probability measure $v$ on $T$, we can write

$$
\mathbb{E}_{\tau_{-i}}\left(\Pi_{i} \mid t_{i}, \beta_{i}\right)=\iint_{T_{-i}} \int_{A_{-i}} \int_{A_{i}} \Pi_{i}(t, a) \beta_{i}\left(t_{i}, d a_{i}\right) \tau_{-i}\left(t_{-i}, d a_{-i}\right) v\left(d t \mid t_{i}\right),
$$

which is equivalent to

$$
\mathbb{E}_{\tau_{-i}}\left(\Pi_{i} \mid t_{i}, \beta_{i}\right)=\iint_{T_{-i}} \int_{A_{-i}} \int_{A_{i}} \Pi_{i}(t, a) \beta_{i}\left(t_{i}, d a_{i}\right) \tau_{-i}\left(t_{-i}, d a_{-i}\right) g_{i}\left(t_{i}\right)\left(t_{-i}\right) \mu_{-i}\left(d t_{-i}\right) .
$$

Thus, the analysis for games with independent types can be equally applied to games with diffuse information. From now on we assume that $f \equiv 1$, and types are not correlated. 


\section{Behavioral perfect Bayesian-Nash equilibrium}

In this section we define perfect BNE for Bayesian games, and discuss its properties, using the list of requirements for refinements formulated by Kohlberg and Mertens as a guide.

Definition 7. A strategy profile $\beta=\left(\beta_{1}, \ldots, \beta_{n}\right)$ is called perfect, if for every player $i$ there exists a set $S_{i} \in \mathcal{T}_{i}$ with $\mu_{i}\left(S_{i}\right)=0$ and a sequence of profiles of completely mixed strategies $\left(\beta^{k}\right)_{k=1}^{\infty}=\left(\beta_{1}^{k}, \ldots, \beta_{n}^{k}\right)_{k=1}^{\infty}$ with the following properties for every player $i$ and every type $t_{i} \in T_{i} \backslash S_{i}$ :

(1) $\lim _{k \rightarrow \infty} \rho^{w}\left(\beta_{i}^{k}\left(t_{i}, \cdot\right), \beta_{i}\left(t_{i}, \cdot\right)\right)=0$,

(2) $\lim _{k \rightarrow \infty} \rho^{w}\left(\beta_{i}^{k}\left(t_{i}, \cdot\right), B R_{i}\left(t_{i}, \beta^{k}\right)\right)=0$

A strategy profile $\beta$ is called a perfect BNE if $\beta$ is both perfect and a BNE. ${ }^{6}$

\subsection{KM requirements}

In their seminal paper, Kohlberg and Mertens (1986) argued that any reasonable refinement of Nash equilibrium should satisfy at least a few self-evident requirements. They proposed a list of such requirements, and searched for a solution concept that satisfied all their requirements. Although in their original paper they did not realize this ambition, later Mertens (1989, 1991) did exactly that.

The list of requirements proposed by Kohlberg and Mertens, with a few minor later amendments incorporated into it, is as follows. We give a brief description of each requirement.

[1] Existence. Every game has at least one solution.

[2] Connectedness. Each solution is a connected set of strategy profiles (possibly a singleton).

[3] Admissibility. Each solution assigns exclusively admissible strategy profiles.

[4] Iterated dominance. Each solution remains to be a solution in the game that results from deletion of a pure strategy that is not admissible.

[5] Backwards induction. Every solution contains a proper equilibrium.

[6] Forward induction. Given a solution, and a pure strategy that is not a best response against any of the profiles assigned by the solution. Then the solution remains to be a solution when this strategy is deleted.

[7] Ordinality. Solutions should be invariant under addition and deletion of payoff-equivalent strategies. Solutions should only depend on the admissible best response correspondence.

We want to test our notion of perfect BNE against these requirements. This agenda has two prime components. First, the above requirements were originally phrased in the context of finite games in strategic form. It is not always immediately clear that any given requirement makes just as much sense outside that framework. We analyze what remains of these requirements within the larger class of Bayesian games.

Second, for those requirements that can reasonably be generalized to the larger class of Bayesian games, we test whether or not perfect BNE satisfies the condition at hand.

We treat each requirement in some detail. We specifically focus on Existence, Admissibility, Iterated dominance, and Ordinality. The remaining requirements are then briefly discussed in a separate paragraph. We conclude this section with a characterization of perfect BNE on a class of Bayesian games defined by two fairly mild conditions.

\subsection{Existence}

We show that Bayesian games with at most countable type spaces, finite action spaces, and continuous payoffs admit a perfect BNE. We also argue that this is more or less all we can hope for. It is known from earlier results by, among others, Simon (2003) and Hellman and Levy (2013), that beyond the restriction of countable type spaces, BNE-and hence also perfect BNE-may not exist.

EXISTENCE We consider a Bayesian game in which each player has a countable type space, a finite action space, and a continuous payoff function. Such a Bayesian game admits a BNE, in view of part (I) of Theorem 1 in Hellman and Levy (2013). In the next theorem we show that such a Bayesian game even admits a perfect BNE.

\footnotetext{
${ }^{6}$ The choice for the weak metric in this definition is mainly inspired by the work of Simon and Stinchcombe's (1995). One could also take a different metric here, but this would largely influence the results. For example, convergence with respect to the uniform metric would be very demanding, resulting in a stronger notion of perfection.
} 
Theorem 8. Let $G$ be a Bayesian game in which every player has a type space that is at most countable, a finite action space, and a continuous payoff function. Then, the game $G$ admits a perfect BNE.

Proof. The proof consists of a number of intuitive steps, and therefore we only provide a sketch. Let $G$ be a Bayesian game as in the theorem. Suppose, without loss of generality, that the type space is $T_{i}=\mathbb{N}$ for every player $i \in N$.

Let $\ell \in \mathbb{N}$ be such that $\ell \geq \max _{i \in N}\left|A_{i}\right|$. For every player $i \in \mathbb{N}$, let $\Delta^{\ell}\left(A_{i}\right)$ denote those mixed actions that place probability at least $\frac{1}{\ell}$ on each action in $A_{i}$. Choose a mixed action $\sigma_{i}^{*} \in \Delta^{\ell}\left(A_{i}\right)$ for every player $i \in \mathbb{N}$.

For every $m \in \mathbb{N}$, let $\Phi_{i}^{m, \ell}$ denote the set of all behavior strategies $\beta_{i}$ of player $i$ such that (1) $\beta\left(t_{i}, \cdot\right) \in \Delta^{\ell}\left(A_{i}\right)$ for every type $t_{i} \in\{1, \ldots, m\}$, and (2) $\beta_{i}\left(t_{i}, \cdot\right)=\sigma_{i}^{*}$ for every type $t_{i} \in \mathbb{N} \backslash\{1, \ldots, m\}$. The set $\Phi_{i}^{m, \ell}$ can be identified with $\times_{t_{i}=1}^{m} \Delta^{\ell}(A) \times \times_{t_{i}=m+1}^{\infty}\left\{\sigma_{i}^{*}\right\}$. Thus, the game in which players are restricted to this space of strategy profiles can be seen as a Bayesian game with finite type spaces and finite action spaces, where the extreme points of the restricted strategy space function as pure actions in the new game. And since Bayesian games with finitely many types and actions admit a BNE, it follows that there is a BNE $\beta^{m, \ell}$ in the game in which every player $i \in N$ is restricted to the strategy set $\Phi_{i}^{m, \ell}$.

For every player $i \in N$, let $\Phi_{i}^{\ell}$ denote the set of all behavior strategies $\beta_{i}$ of player $i$ such that $\beta\left(t_{i}, \cdot\right) \in \Delta^{\ell}\left(A_{i}\right)$ for every type $t_{i} \in \mathbb{N}$. The set $\Phi_{i}^{\ell}$ can be identified with $\times_{t_{i}=1}^{\infty} \Delta^{\ell}(A)$, which is compact. Therefore, the sequence $\beta^{m, \ell}$, as $m \rightarrow \infty$, has an accumulation point $\beta^{\ell}$ in $\times_{i \in N} \Phi_{i}^{\ell}$. By continuity of the payoff functions, $\beta^{\ell}$ is a BNE in the game in which every player $i \in N$ is restricted to the strategy set $\Phi_{i}^{\ell}$.

The set of behavior strategies of player $i$ can be identified with $\times_{t_{i}=1}^{\infty} \Delta(A)$, which is also compact. Therefore, we may assume that the sequence $\beta^{\ell}$ converges to a strategy profile $\beta$. Due to continuity, $\beta$ is a BNE in the game G. Clearly, condition (1) of Definition 7 is satisfied.

Also, given a type $t_{i}$ of player $i$, the set $A_{i}$ of pure strategies at the disposal of type $t_{i}$ is finite. Thus, given the type $t_{i}$, we can take a subsequence so that condition (2) of Definition 7

(2) $\lim _{\ell \rightarrow \infty} \rho^{w}\left(\beta_{i}^{\ell}\left(t_{i}, \cdot\right), B R_{i}\left(t_{i}, \beta^{\ell}\right)\right)=0$

holds. So, since there are only countably many types, we can use a diagonal argument to ensure that (2) holds for every $i \in N$ and every $t_{i} \in T_{i}$. Hence, the strategy profile $\beta$ is perfect.

NON-EXISTENCE It is difficult to obtain existence results beyond the above conditions. This is highlighted by part (II) of Theorem 1 in Hellman and Levy (2013), who provide examples of Bayesian games in which players have type spaces with the cardinality of the continuum without a BNE. We also refer to Simon (2003) for Bayesian games without BNE.

Perfect BNE may also fail to exist for other reasons. For instance, in Example 5 a perfect strategy profile, and therefore a perfect BNE, does not exist, because the best response set in this game is always empty. An example of a perfect strategy profile which is not a BNE is given in the following example.

Example 9. Consider the following Bayesian game with only one player: $T_{1}=\left\{t_{1}\right\}, A_{1}=[0,1], \Pi_{1}\left(t_{1}, x\right)=1$ for every $x \in[0,1)$, and $\Pi_{1}\left(t_{1}, 1\right)=0$. Define strategies $\beta_{1}$ and $\beta_{1}^{k}$, for every $k \in \mathbb{N}$, as follows: $\beta_{1}\left(t_{1}, \cdot\right)=\delta_{1}(\cdot)$ and $\beta_{1}^{k}\left(t_{1}, \cdot\right)=(1-$ $\left.\frac{1}{k}\right) \delta_{1-\frac{1}{k}}(\cdot)+\frac{1}{k} \sigma(\cdot)$, for every $k \in \mathbb{N}$, where $\delta_{x}$ is the Dirac measure on action $x$ and $\sigma$ is the uniform distribution on the Borel sets of $[0,1]$. Note that the set of the pure best responses (that is, optimal strategies) of player 1 consists of all actions in $[0,1)$. So, the strategy $\beta_{1}$ and the sequence of completely mixed strategies $\left(\beta_{1}^{k}\right)_{k=1}^{\infty}$ satisfy conditions (1) and (2) of Definition 7. Therefore, $\beta_{1}$ is a perfect strategy profile, but it is clearly not a BNE.

Remarks. The above example and all further examples with a finite type space can be generalized to a game with an infinite type space. Indeed, suppose that player $i$ has a finite type space $T_{i}=\left\{t_{i}^{1}, \ldots, t_{i}^{n}\right\}$. Then, take $n$ mutually disjoint nonempty and closed intervals $Z^{1}, \ldots, Z^{n}$, and change the type space of player $i$ to $\widetilde{T}_{i}=Z^{1} \cup \ldots \cup Z^{n}$. The idea is that each type in the interval $Z^{j}$ is a copy of type $t_{i}^{j}$. Thus, define the prior probability measure $\widetilde{\mu}_{i}$ on $\widetilde{T}_{i}$ such that $\tilde{\mu}_{i}\left(Z^{j}\right)=\mu_{i}\left(t_{i}^{j}\right)$ for each $j=1, \ldots, n$. Finally, for each player, define a new payoff function in a natural way. In the new game, player $i$ has infinitely many types. This can of course be repeated for each player who has only finitely many types.

\subsection{Admissibility}

In general, even in games with complete information (i.e. when the type spaces are singletons), admissibility in the sense of Kohlberg and Mertens no longer holds. This was already demonstrated by Example 2.1 in Simon and Stinchcombe (1995). The following example again stresses this observation.

WEAKLY DOMINATED STRATEGIES This is an example of a game with a unique Nash equilibrium, in which both players play a weakly dominated strategy. Example 2.1 of Simon and Stinchcombe is a similar example with interval action spaces and continuous payoffs. In our example, action spaces are countably infinite and compact, payoffs are also continuous. 
Example 10. The action spaces are $A_{1}=A_{2}=\{1,2,3, \ldots, \infty\}$, where all natural numbers are isolated points, and $\infty$ is the limit of the sequence $1,2, \ldots$ The topology is shown in the picture below:

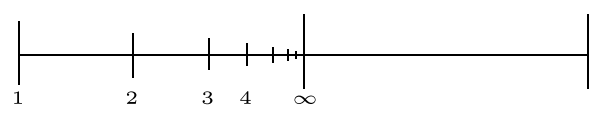

The payoff functions $u_{1}$ and $u_{2}$ are symmetric and $u_{1}$ is given in the table below. Player 1 is the row player and player 2 the column player.

\begin{tabular}{|c|cccccc|}
\hline$u_{1}$ & 1 & 2 & 3 & 4 & $\ldots$ & $\infty$ \\
\hline 1 & 0 & 0 & 0 & 0 & $\ldots$ & 0 \\
2 & 1 & $\frac{-1}{8}$ & 0 & 0 & $\ldots$ & 0 \\
3 & 0 & $\frac{1}{2}$ & $\frac{-1}{16}$ & 0 & $\ldots$ & 0 \\
4 & 0 & 0 & $\frac{1}{4}$ & $\frac{-1}{32}$ & $\ldots$ & 0 \\
$\vdots$ & $\vdots$ & $\vdots$ & $\vdots$ & $\vdots$ & $\ddots$ & $\vdots$ \\
$\infty$ & 0 & 0 & 0 & 0 & $\ldots$ & 0 \\
\hline
\end{tabular}

Notice that the pure action $\infty$ is weakly dominated by $\left(0, \frac{1}{2}, \frac{1}{4}, \frac{1}{8}, \ldots, 0\right)$.

Claim. The strategy pair $(\infty, \infty)$ is the unique Nash equilibrium in this game.

Proof. It is obviously an equilibrium. We argue it's the only one in this game. Let the strategy pair ( $p, q)$ with $p=$ $\left(p_{1}, p_{2}, \ldots, p_{\infty}\right)$ and $q=\left(q_{1}, q_{2}, \ldots, q_{\infty}\right)$ be a Nash equilibrium. We show that $p=q=\infty$ (so, $\left.p_{\infty}=q_{\infty}=1\right)$.

Let $v_{1}$ and $v_{2}$ be the payoffs to player 1 and 2 resp. when the pair $(p, q)$ is played. We consider two cases.

Case 1. If $v_{1} \leq 0$. Suppose that $q \neq \infty$. Then $q_{k}>0$ for some $k=1,2, \ldots$ Since for player 1 playing strategy $k+1$ should yield non-positive payoff, we get that

$$
\frac{1}{2^{k-1}} \cdot q_{k}-\frac{1}{2^{k+2}} \cdot q_{k+1} \leq 0,
$$

which yields $8 \cdot q_{k} \leq q_{k+1}$. In particular $q_{k+1}>0$ so that the argument can be iterated: $8 \cdot q_{k+1} \leq q_{k+2}, 8 \cdot q_{k+2} \leq q_{k+3}$, etc. Contradiction.

This shows that necessarily $q=\infty$, so that $v_{2}=0$. The symmetric argument then gives $p=\infty$ as well, and so that $v_{1}=0$.

Case 2. If $v_{1}>0$ and $v_{2}>0$. Since $v_{1}>0$, it follows that $p_{1}=0$. In the same way, $q_{1}=0$. Then, since $q_{1}=0$ and $v_{1}>0$, also $p_{2}=0$. etc. We find that $p=q=\infty$, which contradicts $v_{1}>0$.

All in all, the only Nash equilibrium is $(\infty, \infty)$.

LIMIT UNDOMINATEDNESS Limit undominatedness is one of the few watered down interpretations of admissibility that remain valid outside the context of finite strategic form games. Since every limit undominated strategy profile is also limit admissible, as per definition of limit admissibility in Simon and Stinchcombe (1995), the theorem applies to limit admissibility too.

A probability measure $\sigma_{i}$ on $A_{i}$ is said to dominate a probability measure $\sigma_{i}^{\prime}$ on $A_{i}$ for a type $t_{i} \in T_{i}$, if

$$
\mathbb{E}_{\tau_{-i}}\left(\Pi_{i} \mid t_{i}, \sigma_{i}\right) \geq \mathbb{E}_{\tau_{-i}}\left(\Pi_{i} \mid t_{i}, \sigma_{i}^{\prime}\right)
$$

holds for every strategy profile $\tau_{-i}$ and the strict inequality holds for at least one $\tau_{-i}$. A probability measure $\sigma_{i}$ is called undominated for a type $t_{i}$ if there is no probability measure on $A_{i}$ that dominates $\sigma_{i}$ for $t_{i}$. 
A strategy $\beta_{i}$ is called limit undominated if there is a set $S_{i} \in \mathcal{T}_{i}$ with $\mu_{i}\left(S_{i}\right)=0$ such that for every $t_{i} \in T_{i} \backslash S_{i}$ there is a sequence of undominated probability measures $\left(\sigma_{i}^{k}\right)_{k=1}^{\infty}$ on the action space $A_{i}$ for which we have $\rho^{w}\left(\beta_{i}\left(t_{i}, \cdot\right), \sigma_{i}^{k}\right) \rightarrow 0$ as $k \rightarrow \infty$. A strategy profile $\beta=\left(\beta_{1}, \ldots, \beta_{n}\right)$ is called limit undominated if $\beta_{i}$ is limit undominated for every player $i$.

In the following theorem we show that a perfect BNE is limit undominated, provided that the payoff function of each player, given the types, is continuous in actions.

Theorem 11. Let $\beta=\left(\beta_{1}, \ldots, \beta_{n}\right)$ be a perfect $B N E$. If $\Pi_{i}(t, \cdot): A \rightarrow \mathbb{R}$ is continuous for every type-vector $t \in T$ and every player $i$, then $\beta$ is limit undominated.

Proof. Let $\beta=\left(\beta_{1}, \ldots, \beta_{n}\right)$ be a perfect BNE. Then, according to Definition 7 , for every player $i$ there is a set $S_{i} \in \mathcal{T}_{i}$ with $\mu_{i}\left(S_{i}\right)=0$ such that for every $t_{i} \in T_{i} \backslash S_{i}$ and a sequence of profiles of completely mixed strategies $\left(\beta^{k}\right)_{k=1}^{\infty}=\left(\beta_{1}^{k}, \ldots, \beta_{n}^{k}\right)_{k=1}^{\infty}$ we have

$$
\begin{aligned}
& \lim _{k \rightarrow \infty} \rho^{w}\left(\beta_{i}^{k}\left(t_{i}, \cdot\right), \beta_{i}\left(t_{i}, \cdot\right)\right)=0, \\
& \lim _{k \rightarrow \infty} \rho^{w}\left(\beta_{i}^{k}\left(t_{i}, \cdot\right), B R_{i}\left(t_{i}, \beta^{k}\right)\right)=0 .
\end{aligned}
$$

Take an arbitrary player $i$, and fix a type $t_{i} \in T_{i} \backslash S_{i}$. From condition (2), there exists a sequence of probability measures $\left(\sigma_{i}^{k}\right)_{k=1}^{\infty}$ on $A_{i}$ such that $\sigma_{i}^{k} \in B R_{i}\left(t_{i}, \beta^{k}\right)$, for every $k$, and $\rho^{w}\left(\beta_{i}^{k}\left(t_{i}, \cdot\right), \sigma_{i}^{k}\right) \rightarrow 0$ as $k \rightarrow \infty$. By the triangle inequality, $\rho^{w}\left(\sigma_{i}^{k}, \beta_{i}\left(t_{i}, \cdot\right)\right) \rightarrow 0$ as $k \rightarrow \infty$. Since $\sigma_{i}^{k}$ is a best response against the completely mixed profile $\beta^{k}$, for every $k$, it follows from Lemma A.2 in Bajoori et al. (2013) that $\sigma_{i}^{k}$ is undominated, for every $k^{7}$ Hence $\beta_{i}$ is limit undominated.

Remark. The above theorem assumes that the payoff functions, given the types, are continuous in actions. In fact we only need the following weaker condition: for every player $i$ and type $t_{i}$, if a probability measure $\tau_{i} \in \Delta\left(A_{i}\right)$ dominates a probability measure $\tau_{i}^{\prime} \in \Delta\left(A_{i}\right)$, then the set $\left\{a_{-i} \in A_{-i} \mid \mathbb{E}_{a_{-i}}\left(\Pi_{i} \mid t_{i}, \tau_{i}\right)>\mathbb{E}_{a_{-i}}\left(\Pi_{i} \mid t_{i}, \tau_{i}^{\prime}\right)\right\}$ has a nonempty interior.

\subsection{Iterated dominance}

Iterated dominance, as it was formulated by Kohlberg and Mertens, stipulates that a refinement should be robust against (iterated) deletion of weakly dominated strategies. Example 10 shows that, outside the context of finite strategic form games, iterated deletion of weakly dominated strategies is too much to hope for. Note that, if we eliminate for example $p=\infty$ from the game in Example 10, the result is a game that (1) does no longer have compact action spaces, and (2) does not have a Nash equilibrium. Insisting on robustness of a refinement to such quite drastic changes of the game seems too much to ask of any reasonable solution concept.

ITERATED DELETION OF STRICTLY DOMINATED ACTIONS An action $a_{i} \in A_{i}$ is strictly dominated when for each $t_{i}$ there is a strategy $\sigma_{i} \in \Delta\left(A_{i}\right)$, such that for every strategy profile $\tau_{-i}$,

$$
\mathbb{E}_{\tau_{-i}}\left(\Pi_{i} \mid t_{i}, \sigma_{i}\right)>\mathbb{E}_{\tau_{-i}}\left(\Pi_{i} \mid t_{i}, a_{i}\right)
$$

The requirement that $a_{i}$ is strictly dominated for each type is necessary, since deletion of $a_{i}$ implies that $a_{i}$ is no longer available for any type $t_{i}$. Thus, no type should consider this to be problematic. Hence our requirement.

It is tempting to believe that a perfect BNE will remain a perfect BNE after deletion of a strictly dominated pure action. At least, a BNE will certainly remain a BNE under this operation. And although the claim for perfect BNE sounds plausible, it's not true, not even for perfect Nash equilibrium in finite strategic form games, as the following example shows.

Example 12. Consider the bimatrix game with payoff matrices

$$
A=\left[\begin{array}{cc}
0 & 0 \\
0 & 0 \\
-1 & -1
\end{array}\right] \text { and } B=\left[\begin{array}{ll}
1 & 1 \\
1 & 0 \\
0 & 1
\end{array}\right]
$$

In this game the Nash equilibrium $((1,0,0)(0,1))$ is perfect. It is the limit of the $\varepsilon$-perfect strategy pairs $((1-2 \varepsilon, \varepsilon, \varepsilon)(\varepsilon$, $1-\varepsilon)$ ). However, when we delete the third row, which is strictly dominated for player 1 , the resulting game is

$$
C=\left[\begin{array}{ll}
0 & 0 \\
0 & 0
\end{array}\right] \text { and } D=\left[\begin{array}{ll}
1 & 1 \\
1 & 0
\end{array}\right]
$$

in which game player 2 plays $(1,0)$ in any perfect Nash equilibrium.

\footnotetext{
7 Lemma A.2 in Bajoori et al. (2013) has the following claim for a game of complete information: Provided that the payoff function $u_{i}$ is continuous, if a strategy $\tau_{i} \in \Delta\left(A_{i}\right)$ dominates another strategy $\tau_{i}^{\prime} \in \Delta\left(A_{i}\right)$, then $u_{i}\left(\tau_{i}, \sigma_{-i}\right)>u_{i}\left(\tau_{i}^{\prime}, \sigma_{-i}\right)$ holds for any completely mixed strategy profile $\sigma \in \Delta(A)$. Consequently, any best response to a completely mixed strategy profile is undominated.
} 
Thus, even for finite strategic form games, iterated dominance typically agrees with stronger notions of stability, but not with perfect equilibrium. ${ }^{8}$

We can prove that any BNE puts zero weight on a strictly dominated pure action.

Lemma 13. Let $\beta$ be a BNE. Suppose that $a_{i} \in A_{i}$ is strictly dominated. Then for every type $t_{i} \in T_{i}, \beta_{i}\left(t_{i},\left\{a_{i}\right\}\right)=0$.

Proof. Fix type $t_{i}$. Suppose that $\beta_{i}\left(t_{i},\left\{a_{i}\right\}\right)>0$. We derive a contradiction. Since $a_{i}$ is strictly dominated, there is a strategy $\sigma_{i} \in \Delta\left(A_{i}\right)$ such that

$$
\mathbb{E}_{\beta_{-i}}\left(\Pi_{i} \mid t_{i}, \sigma_{i}\right)>\mathbb{E}_{\beta_{-i}}\left(\Pi_{i} \mid t_{i}, a_{i}\right) .
$$

Define $B_{i}=A_{i} \backslash\left\{a_{i}\right\}, \eta_{i}=\beta_{i}\left(t_{i},\left\{a_{i}\right\}\right)$, and $\tau_{i}=\mathbb{I}_{B_{i}} \cdot \beta_{i}+\eta_{i} \cdot \sigma_{i}$ where $\mathbb{I}_{B_{i}}$ denotes the characteristic function on $B_{i}$. Then

$$
\mathbb{E}_{\beta_{-i}}\left(\Pi_{i} \mid t_{i}, \tau_{i}\right)>\mathbb{E}_{\beta_{-i}}\left(\Pi_{i} \mid t_{i}, \beta_{i}\right),
$$

and $\beta$ is not a BNE. Contradiction.

With a bit more work, one can also show that the restriction of a BNE to the game where the strictly dominated strategy is deleted remains a BNE. This is fairly straightforward though, and somewhat outside the scope of the current paper, so we will not go into details.

\subsection{Ordinality}

While perfect equilibrium satisfies invariance in the setting of finite strategic form games-see for example Mertens (2003) and Vermeulen and Jansen (1997)-perfection violates invariance even in games with complete information if the action spaces are infinite, as is pointed out in Bajoori et al. (2013).

\subsection{Remaining requirements}

CONNECTEDNESS It is well-known, even in the classical setting of finite strategic form games, that the set of perfect equilibria need not be connected. Thus, also in our context, this requirement is not valid for perfect BNE.

BACKWARDS INDUCTION The intuition for forward and backwards induction originate from extensive form games. In that sense these notions do not directly apply to our setting. One more demanding interpretation of Kohlberg and Mertens of backwards induction is that each solution set should contain a proper equilibrium. As it is, proper BNE has not (yet) been defined for Bayesian games. Of course, any possible definition in the spirit of perfect BNE would result in the observation the set of perfect BNE's will contain a proper BNE, as soon as a proper BNE exists.

FORWARD INDUCTION The interpretation of Kohlberg and Mertens of forward induction in the context of finite strategic form games is already violated by perfect Nash equilibrium. This can be seen in Example 12, which features a special case of this requirement. Hence, also perfect BNE fails on this criterion.

\subsection{A characterization}

In this paragraph we provide an alternative definition for perfect BNE on a class of Bayesian games that is specified by the following two measurability conditions:

- Condition M1: For any player $i$ and any strategies $\beta_{i}^{1}, \beta_{i}^{2}$ of player $i$, the map

$$
t_{i} \mapsto \rho^{w}\left(\beta_{i}^{1}\left(t_{i}, \cdot\right), \beta_{i}^{2}\left(t_{i}, \cdot\right)\right)
$$

is measurable.

- Condition M2: For any player $i$, any strategy $\beta_{i}$ of player $i$, and any strategy profile $\tau$, the map

$$
t_{i} \mapsto \rho^{w}\left(\beta_{i}\left(t_{i}, \cdot\right), B R_{i}\left(t_{i}, \tau\right)\right)
$$

is measurable.

\footnotetext{
${ }^{8}$ In fact this observation was one of the key arguments in the motivation of Kohlberg and Mertens to search for set-valued solution concepts. Still, BNE satisfies iterated deletion of strictly dominated strategies, even when interpreted as a singleton-valued solution concept. This particular research topic is outside the scope of the current paper though, and we will not venture into details.
} 
Condition M1 is mild, and M1 is always satisfied if the action spaces are separable (cf. Lemma 32). It seems likely that M2 holds under the assumption that the action spaces are measurable subsets of a Polish (complete and separable metric) space. ${ }^{9}$

Now, we present a number of conditions that are all equivalent to perfection of strategy profiles, given assumptions of M1 and M2. All implications are straightforward, so the formal proof is omitted.

Claim 14. Consider a Bayesian game which satisfies conditions $M 1$ and $M 2$. Then, for every strategy profile $\beta=\left(\beta_{1}, \ldots, \beta_{n}\right)$, equivalent are:

i. The strategy profile $\beta$ is perfect.

ii. There exists a sequence of profiles of completely mixed strategies $\left(\beta^{k}\right)_{k=1}^{\infty}=\left(\beta_{1}^{k}, \ldots, \beta_{n}^{k}\right)_{k=1}^{\infty}$ such that for every player $i$ and every $\varepsilon>0$ :

(ii.1) $\lim _{k \rightarrow \infty} \mu_{i}\left\{t_{i} \in T_{i} \mid \rho^{w}\left(\beta_{i}^{k}\left(t_{i}, \cdot\right), \beta_{i}\left(t_{i}, \cdot\right)\right) \geq \varepsilon\right\}=0$,

(ii.2) $\lim _{k \rightarrow \infty} \mu_{i}\left\{t_{i} \in T_{i} \mid \rho^{w}\left(\beta_{i}^{k}\left(t_{i}, \cdot\right), B R_{i}\left(t_{i}, \beta^{k}\right)\right) \geq \varepsilon\right\}=0$.

iii. There exists a sequence of profiles of completely mixed strategies $\left(\beta^{k}\right)_{k=1}^{\infty}=\left(\beta_{1}^{k}, \ldots, \beta_{n}^{k}\right)_{k=1}^{\infty}$ such that for every player $i$ :

$$
\begin{aligned}
& \text { (iii.1) } \lim _{k \rightarrow \infty} \int_{T_{i}} \rho^{w}\left(\beta_{i}^{k}\left(t_{i}, \cdot\right), \beta_{i}\left(t_{i}, \cdot\right)\right) \mu_{i}\left(d t_{i}\right)=0, \\
& \text { (iii.2) } \lim _{k \rightarrow \infty} \int_{T_{i}} \rho^{w}\left(\beta_{i}^{k}\left(t_{i}, \cdot\right), B R_{i}\left(t_{i}, \beta^{k}\right)\right) \mu_{i}\left(d t_{i}\right)=0 .
\end{aligned}
$$

The intuition behind conditions (ii) and (iii) is the following. For every player $i$ and every $k \in \mathbb{N}$, define a function $X_{i}^{k}: T_{i} \rightarrow \mathbb{R}$ by $X_{i}^{k}\left(t_{i}\right)=\rho^{w}\left(\beta_{i}^{k}\left(t_{i}, \cdot\right), \beta_{i}\left(t_{i}, \cdot\right)\right)$, and a function $Y_{i}^{k}: T_{i} \rightarrow \mathbb{R}$ by $Y_{i}^{k}\left(t_{i}\right)=\rho^{w}\left(\beta_{i}^{k}\left(t_{i}, \cdot\right), B R_{i}\left(t_{i}, \beta^{k}\right)\right)$. Due to the assumptions M1 and M2, these functions are measurable, and therefore they are random variables on the measurable space $\left(T_{i}, \mathcal{T}_{i}\right)$. Conditions (ii.1) and (ii.2) respectively require that both sequences $X_{i}^{k}$ and $Y_{i}^{k}$ converge in probability to zero (i.e. to the random variable that is zero everywhere). So, the probability that $X_{i}^{k}$ and $Y_{i}^{k}$ are far from zero becomes negligible for large $k$. Conditions (iii.1) and (iii.2) mean that both sequences $X_{i}^{k}$ and $Y_{i}^{k}$ converge in expectation to zero, with respect to the distribution $\mu_{i}$. This makes intuitive sense because the type of player $i$ is drawn from $T_{i}$ according to $\mu_{i}$.

\section{Alternative notions of perfection}

In this section, we examine two alternative notions of perfection, where convergence on the set of types is required to hold pointwise for each type or, respectively, uniformly for all types. ${ }^{10}$

\subsection{Pointwise perfection}

Definition 15. A strategy profile $\beta=\left(\beta_{1}, \ldots, \beta_{n}\right)$ is called pointwise-perfect, if there exists a sequence of profiles of completely mixed strategies $\left(\beta^{k}\right)_{k=1}^{\infty}=\left(\beta_{1}^{k}, \ldots, \beta_{n}^{k}\right)_{k=1}^{\infty}$ with the following properties for every player $i$ and each type $t_{i} \in T_{i}$ :

(1) $\lim _{k \rightarrow \infty} \rho^{w}\left(\beta_{i}^{k}\left(t_{i}, \cdot\right), \beta_{i}\left(t_{i}, \cdot\right)\right)=0$,

(2) $\lim _{k \rightarrow \infty} \rho^{w}\left(\beta_{i}^{k}\left(t_{i}, \cdot\right), B R_{i}\left(t_{i}, \beta^{k}\right)\right)=0$

A strategy profile $\beta$ is called a pointwise-perfect BNE if $\beta$ is a BNE and pointwise-perfect.

Note that conditions (1) and (2) in the above definition require pointwise convergence of the corresponding distances over the set of possible types. Hence, every pointwise-perfect strategy profile is perfect, and every pointwise-perfect BNE is a perfect BNE.

The following theorem provides sufficient conditions under which a pointwise-perfect strategy profile is a BNE. The proof can be found in Appendix I.

\footnotetext{
9 This condition for M2 is suggested by one of the referees.

10 In game theory, sets of measure 0 often play an unimportant role. In extensive form games, however, various solution concepts take information sets into account that are reached with probability 0 . In the definitions of this section, we take information sets into account that are only reached with probability 0 with respect to the prior probability distribution on the type spaces of the players.
} 
Theorem 16. In a Bayesian game where the action spaces are separable and $\Pi_{i}(t, \cdot)$ is continuous on $A$ for every player $i$ and every $t \in T$, every pointwise-perfect strategy profile is a BNE.

It is clear that every pointwise-perfect BNE is perfect. The following example proves that the converse is not always true.

Example 17. Consider the following Bayesian game with two players: The type spaces are $T_{1}=\left\{t_{1}\right\}$ and $T_{2}=\left\{t_{2}^{1}, t_{2}^{2}\right\}$, and $\mu_{2}$ is given by $\mu_{2}\left(t_{2}^{1}\right)=1$ and $\mu_{2}\left(t_{2}^{2}\right)=0$. The action spaces are $A_{1}=\{U, D\}$ and $A_{2}=\{L, R\}$. The payoff matrix is the following if player 1 is given type $t_{1}$ whereas player 2 is given type $t_{2}^{m}$ for $m=1,2$ :

\begin{tabular}{r|rr}
$\left(t_{1}, t_{2}^{m}\right)$ & $L$ & $R$ \\
\hline$U$ & 0,1 & 0,0 \\
$D$ & 1,0 & 1,0
\end{tabular}

Define $p_{1}\left(t_{1}\right)=D, p_{2}\left(t_{2}^{1}\right)=L$ and $p_{2}\left(t_{2}^{2}\right)=R$. Let $\beta_{1}$ and $\beta_{2}$ be the deterministic strategies corresponding to $p_{1}$ and $p_{2}$ respectively. It is clear that $\left(\beta_{1}, \beta_{2}\right)$ is a perfect BNE, by choosing $S_{1}=\emptyset$ and $S_{2}=\left\{t_{2}^{2}\right\}$ in Definition 7. Yet, $\left(\beta_{1}, \beta_{2}\right)$ is not pointwise-perfect, because player 2 chooses $R$ with probability 1 if he receives type $t_{2}^{2}$.

We remark that a similar example can be made where $T_{2}=[0,1], \mu_{2}$ is the uniform distribution on $T_{2}$, and where the strategy profile is not pointwise-perfect only due to a single type, say type $t_{2}=1$.

The following theorem presents conditions under which there is a strong connection between perfection and pointwiseperfection. The proof can be found in Appendix I.

Proposition 18. Let $\beta$ be a perfect BNE in a Bayesian game with compact action spaces. Let $S_{i}$, for every player $i$, and $\left(\beta^{k}\right)_{k=1}^{\infty}$ be as in Definition 7 for $\beta$. Suppose that $S_{i}$ is countable for every player $i$ and $B R_{i}\left(t_{i}, \beta^{k}\right)$ is nonempty for every player $i$, every type $t_{i} \in S_{i}$ and every $k \in \mathbb{N}$. Then, there is a pointwise-perfect BNE $\hat{\beta}$ such that $\hat{\beta}_{i}\left(t_{i}, \cdot\right)=\beta_{i}\left(t_{i}, \cdot\right)$ holds for every player $i$ and every type $t_{i} \in T_{i} \backslash S_{i}$.

The following corollary follows easily from Proposition 18.

Corollary 19. Consider a Bayesian game in which the type spaces are countable, the action spaces are compact, and the payoff functions are continuous. Then, for every perfect BNE $\beta$, there exists a pointwise-perfect BNE $\hat{\beta}$ such that, for every player $i$, we have $\hat{\beta}_{i}\left(t_{i}, \cdot\right)=$ $\beta_{i}\left(t_{i}, \cdot\right)$ for $\mu_{i}$-a.e. type $t_{i} \in T_{i}$.

The next example shows that the condition that $S_{i}$ is countable is crucial in Proposition 18 if there are at least three players. In this game, the type and action spaces are compact and the payoff functions are continuous. We do not know if this condition is also crucial for games with only two players.

Example 20. Consider the following Bayesian game with three players. The type spaces ${ }^{11}$ are $T_{1}=\{0,1\}^{\mathbb{N}}, T_{2}=\left\{t_{2}\right\}$ and $T_{3}=\left\{t_{3}\right\}$. The metric $d_{T_{1}}$ on $T_{1}$ is defined as follows: for $t_{1}, t_{1}^{\prime} \in T_{1}$, if $t_{1}=t_{1}^{\prime}$ then let $d_{T_{1}}\left(t_{1}, t_{1}^{\prime}\right)=0$, otherwise if $m$ is the first coordinate in which $t_{1}$ and $t_{1}^{\prime}$ differ, then let $d_{T_{1}}\left(t_{1}, t_{1}^{\prime}\right)=2^{-m}$. Notice that $d_{T_{1}}$ induces the product topology on $T_{1}$. Further, let $\mu_{1}=\delta_{(1,1, \ldots)}$, i.e. the Dirac measure on the type $(1,1, \ldots)$. The action spaces are $A_{1}=\{U, D\}, A_{2}=$ $\left\{a_{1}, a_{2}, \ldots, a_{\infty}\right\}$, and $A_{3}=\left\{b_{1}, b_{2}, \ldots, b_{\infty}\right\}$, in which $a_{m}=b_{m}=1-\frac{1}{m}$ for every $m \in \mathbb{N}$ and $a_{\infty}=b_{\infty}=1$. For every type $t_{1} \in\{0,1\}^{\mathbb{N}}$, let $f_{m}\left(t_{1}\right)$ be the $m$-th coordinate of the sequence $t_{1}$. When player 1 is given type $t_{1} \in\{0,1\}^{\mathbb{N}} \backslash\{1,1, \ldots\}$, the payoff of player 1 is independent of the action chosen by player 3 , and it is given by

\begin{tabular}{r|cc}
$t_{1} \in\{0,1\}^{\mathbb{N}} \backslash\{(1,1, \ldots)\}$ & $a_{m}$ & $a_{\infty}$ \\
\hline$U$ & $f_{m}\left(t_{1}\right)$ & 0 \\
$D$ & $1-f_{m}\left(t_{1}\right)$ & 0
\end{tabular}

whereas if player 1 is given type $t_{1}=(1,1, \ldots)$ :

11 Such rather abstract type spaces are not unusual, see for instance Simon (2003) or Hellman and Levy (2013), and the references therein. 


$$
\begin{array}{r|cc}
t_{1}=(1,1, \ldots) & a_{m} & a_{\infty} \\
\hline U & 0 & 0 \\
D & 0 & 0
\end{array}
$$

The payoffs of players 2 and 3 are independent of the action chosen by player 1 , and are given by:

\begin{tabular}{c|cccccc} 
& $b_{1}$ & $b_{2}$ & $b_{3}$ & $b_{4}$ & $\ldots$ & $b_{\infty}$ \\
\hline$a_{1}$ & 0,0 & $0, \frac{1}{2}$ & $0, \frac{1}{3}$ & $0, \frac{1}{4}$ & $\ldots$ & 0,0 \\
$a_{2}$ & $\frac{1}{2}, 0$ & 0,0 & $0, \frac{1}{3}$ & $0, \frac{1}{4}$ & $\ldots$ & 0,0 \\
$a_{3}$ & $\frac{1}{3}, 0$ & $\frac{1}{3}, 0$ & 0,0 & $0, \frac{1}{4}$ & $\ldots$ & 0,0 \\
$a_{4}$ & $\frac{1}{4}, 0$ & $\frac{1}{4}, 0$ & $\frac{1}{4}, 0$ & 0,0 & $\ldots$ & 0,0 \\
$\vdots$ & $\vdots$ & $\vdots$ & $\vdots$ & $\vdots$ & $\vdots$ & $\vdots$ \\
$a_{\infty}$ & 0,0 & 0,0 & 0,0 & 0,0 & $\ldots$ & 0,0
\end{tabular}

In general, if player 2 chooses action $a_{m}$ and player 3 chooses action $b_{\ell}$, with $m, \ell \in \mathbb{N}$, then player 2's payoff is $\frac{1}{m}$ if $m>\ell$, and 0 otherwise, and player 3's payoff is $\frac{1}{\ell}$ if $\ell>m$, and 0 otherwise.

Note that the type and action spaces in this game are all compact. Since the payoff functions of players 2 and 3 are continuous, and player 1 has only finitely many actions, the best reply set $B R_{i}\left(t_{i}, \beta\right)$ is nonempty for every player $i$, every $t_{i} \in$ $T_{i}$ and every strategy profile $\beta$. This game has the following properties: (1) there exists a perfect equilibrium in this game, but (2) this game admits no pointwise-perfect strategy profile. The proof of both properties can be found in Appendix I.

\subsection{Uniform perfection}

Definition 21. A strategy profile $\beta=\left(\beta_{1}, \ldots, \beta_{n}\right)$ is called uniform-perfect, if there exists a sequence of profiles of completely mixed strategies $\left(\beta^{k}\right)_{k=1}^{\infty}=\left(\beta_{1}^{k}, \ldots, \beta_{n}^{k}\right)_{k=1}^{\infty}$ with the following properties for every player $i$ :

(1) $\lim _{k \rightarrow \infty} \sup _{t_{i} \in T_{i}} \rho^{w}\left(\beta_{i}^{k}\left(t_{i}, \cdot\right), \beta_{i}\left(t_{i}, \cdot\right)\right)=0$,

(2) $\lim _{k \rightarrow \infty} \sup _{t_{i} \in T_{i}} \rho^{w}\left(\beta_{i}^{k}\left(t_{i}, \cdot\right), B R_{i}\left(t_{i}, \beta^{k}\right)\right)=0$.

A strategy profile $\beta$ is called a uniform-perfect BNE if $\beta$ is a BNE and uniform-perfect.

Note that conditions (1) and (2) in the above definition require a uniform convergence of the corresponding distances over the set of possible types. In the special case when the game has complete information, i.e. each $T_{i}$ is a singleton, our definition coincides with Simon and Stinchcombe's weak perfect equilibrium in Simon and Stinchcombe (1995).

It is clear that every uniform-perfect BNE is also pointwise-perfect. Now, we provide a game which demonstrates that the converse is not always true. This game has a pointwise-perfect BNE, but it has no uniform-perfect BNE, in fact not even a uniform-perfect strategy profile, although the type and action spaces are compact and the payoff functions are continuous.

Example 22. Consider the following Bayesian game with two players: Player 1 has only one possible type, $T_{1}=\left\{t_{1}\right\}$, whereas player 2's type space is $T_{2}=\left\{t_{2}^{1}, t_{2}^{2}, \ldots, t_{2}^{\infty}\right\}$ in which $t_{2}^{\infty}$ is the limit point of the sequence $\left(t_{2}^{m}\right)_{m=1}^{\infty}$. The probability measure $\mu_{2}$ on $\left(T_{2}, \mathcal{T}_{2}\right)$ is arbitrary. ${ }^{12}$ The action spaces are $A_{1}=\{U, D\}$ and $A_{2}=\{L, R\}$. The payoff matrix when player 1 is the row-player and is given type $t_{1}$, and player 2 is the column-player and is given type $t_{2}^{m}$, for every $m \in \mathbb{N}$, is the following:

\begin{tabular}{r|rr}
$\left(t_{1}, t_{2}^{m}\right)$ & $L$ & $R$ \\
\hline$U$ & $0,-\frac{1}{m}$ & 0,0 \\
$D$ & $1, \frac{1}{m^{2}}$ & 1,0
\end{tabular}

12 The more interesting case is when $\mu_{2}$ assigns a positive mass to every point in $T_{2}$; otherwise one would have almost everywhere uniform convergence. 
and the payoff matrix when player 1 is given type $t_{1}$ and player 2 is given type $t_{2}^{\infty}$ is:

\begin{tabular}{r|rr}
$\left(t_{1}, t_{2}^{\infty}\right)$ & $L$ & $R$ \\
\hline$U$ & 0,0 & 0,0 \\
$D$ & 1,0 & 1,0
\end{tabular}

Observe that the type and action spaces in this game are compact, and the payoff functions are continuous.

Define $p_{1}\left(t_{1}\right)=D$ and $p_{2}\left(t_{2}^{m}\right)=L$, for every $m \in \mathbb{N} \cup\{\infty\}$. Let $\beta_{1}$ and $\beta_{2}$ be the deterministic strategies corresponding to $p_{1}$ and $p_{2}$ respectively. We claim that $\left(\beta_{1}, \beta_{2}\right)$ is a pointwise-perfect BNE, but the game has no uniform-perfect strategy profile, so no uniform-perfect BNE either.

The proof that $\left(\beta_{1}, \beta_{2}\right)$ is a pointwise-perfect BNE: Clearly, $\left(\beta_{1}, \beta_{2}\right)$ is a BNE, so it remains to prove that $\left(\beta_{1}, \beta_{2}\right)$ is pointwise-perfect. For every $k \in \mathbb{N}$, define two completely mixed strategies $\beta_{1}^{k}$ and $\beta_{2}^{k}$ by letting $\beta_{1}^{k}\left(t_{1}, \cdot\right)=\left(1-\frac{1}{k}\right) \delta_{D}(\cdot)+$ $\frac{1}{k} \delta_{U}(\cdot)$ and $\beta_{2}^{k}\left(t_{2}^{m}, \cdot\right)=\left(1-\frac{1}{k}\right) \delta_{L}(\cdot)+\frac{1}{k} \delta_{R}(\cdot)$ for every $m \in \mathbb{N} \cup\{\infty\}$, where $\delta_{x}$ denotes the Dirac measure on $x$. The sequence $\left(\beta^{k}\right)_{k=1}^{\infty}=\left(\beta_{1}^{k}, \beta_{2}^{k}\right)_{k=1}^{\infty}$ clearly satisfies condition (1) of Definition 15, and it also satisfies condition (2) for player 1 and condition (2) for type $t_{2}^{\infty}$ of player 2. It remains to verify condition (2) for an arbitrary type $t_{2}^{m}$ for player 2 where $m \in \mathbb{N}$. Take such a type $t_{2}^{m}$. We have

$$
\mathbb{E}_{\beta_{1}^{k}}\left(\Pi_{2} \mid t_{2}^{m}, L\right)=\left(1-\frac{1}{k}\right) \cdot \frac{1}{m^{2}}+\frac{1}{k} \cdot\left(-\frac{1}{m}\right),
$$

which is strictly positive for large $k$. Hence, $B R_{2}\left(t_{2}^{m}, \beta^{k}\right)=\left\{\delta_{L}\right\}$ for large $k$, which implies that condition (2) of Definition 15 holds for type $t_{2}^{m}$. Thus, $\left(\beta_{1}, \beta_{2}\right)$ is pointwise-perfect indeed.

The proof that this game has no uniform-perfect strategy profile: Suppose by way of contradiction that $\tau=\left(\tau_{1}, \tau_{2}\right)$ is a uniform-perfect strategy profile. Then, there is a sequence of completely mixed strategy profiles $\left(\tau^{k}\right)_{k=1}^{\infty}=\left(\tau_{1}^{k}, \tau_{2}^{k}\right)_{k=1}^{\infty}$ that satisfy conditions (1) and (2) of Definition 21 for $\tau$. First notice that, by the triangle inequality for $\rho^{w}$, we have

$$
\begin{aligned}
\sup _{t_{2} \in T_{2}} \rho^{w}\left(\tau_{2}\left(t_{2}, \cdot\right), B R_{2}\left(t_{2}, \tau^{k}\right)\right) & \leq \sup _{t_{2} \in T_{2}}\left[\rho^{w}\left(\tau_{2}^{k}\left(t_{2}, \cdot\right), \tau_{2}\left(t_{2}, \cdot\right)\right)+\rho^{w}\left(\tau_{2}^{k}\left(t_{2}, \cdot\right), B R_{2}\left(t_{2}, \tau^{k}\right)\right)\right] \\
& \leq \sup _{t_{2} \in T_{2}} \rho^{w}\left(\tau_{2}^{k}\left(t_{2}, \cdot\right), \tau_{2}\left(t_{2}, \cdot\right)\right)+\sup _{t_{2} \in T_{2}} \rho^{w}\left(\tau_{2}^{k}\left(t_{2}, \cdot\right), B R_{2}\left(t_{2}, \tau^{k}\right)\right),
\end{aligned}
$$

and therefore conditions (1) and (2) imply

$$
\lim _{k \rightarrow \infty} \sup _{t_{2} \in T_{2}} \rho^{w}\left(\tau_{2}\left(t_{2}, \cdot\right), B R_{2}\left(t_{2}, \tau^{k}\right)\right)=0 .
$$

For every $k \in \mathbb{N}$, the probability measure $\tau_{1}^{k}\left(t_{1}, \cdot\right)$ can be written in the form $\tau_{1}^{k}\left(t_{1}, \cdot\right)=\left(1-\varepsilon_{k}\right) \delta_{U}(\cdot)+\varepsilon_{k} \delta_{D}(\cdot)$ with some $\varepsilon_{k} \in(0,1)$. Due to condition (2) for player 1 , we must have that $\varepsilon_{k} \rightarrow 1$ as $k \rightarrow \infty$.

For every $k \in \mathbb{N}$ and every type $t_{2}^{m}$ for player 2 , where $m \in \mathbb{N}$, we have

$$
\mathbb{E}_{\tau_{1}^{k}}\left(\Pi_{2} \mid t_{2}^{m}, L\right)=\varepsilon_{k} \cdot \frac{1}{m^{2}}+\left(1-\varepsilon_{k}\right) \cdot\left(-\frac{1}{m}\right),
$$

whereas $\mathbb{E}_{\tau_{1}^{k}}\left(\Pi_{2} \mid t_{2}^{m}, R\right)=0$. It has two consequences. First, for every $t_{2}^{m}$, where $m \in \mathbb{N}$, we have $B R_{2}\left(t_{2}^{m}, \tau^{k}\right)=\left\{\delta_{L}\right\}$ for large $k$, which in view of (1) yields $\tau_{2}\left(t_{2}^{m}, \cdot\right)=\delta_{L}(\cdot)$ for every $t_{2}^{m}$, where $m \in \mathbb{N}$. Second, for every $k \in \mathbb{N}$, we have $B R_{2}\left(t_{2}^{m}, \tau^{k}\right)=\left\{\delta_{R}\right\}$ for large $m$. In conclusion, for every $k \in \mathbb{N}$, if $m$ is large, then $\tau_{2}\left(t_{2}^{m}, \cdot\right)=\delta_{L}(\cdot)$ and $B R_{2}\left(t_{2}^{m}, \tau^{k}\right)=\left\{\delta_{R}\right\}$. This is in contradiction with (1), so $\tau$ is not a uniform-perfect strategy profile.

\subsection{Perfection in finite Bayesian games}

In games with finitely many types and actions

[1] it is clear that the uniform and the pointwise approaches coincide, and

[2] by Proposition 18, a perfect BNE is also pointwise-perfect or uniform-perfect, unless certain types occur with probability 0.

Thus, Theorem 8 together with Proposition 18 imply the following result.

Corollary 23. Every Bayesian game with finitely many actions and finitely many types admits a pointwise-perfect (or equivalently, uniform-perfect) BNE. 


\section{A second-price auction with interdependent values}

We examine perfect equilibrium in a second price auction with interdependent values, in which bidders have no dominant strategies. In this auction we identify a symmetric BNE $\beta$, and argue that $\beta$ is a natural candidate solution for the auction from a conceptual and normative perspective. The BNE $\beta$ separates the types, and it is continuous-even differentiable, so that bids increase smoothly with type. We show that $\beta$ is unique in this respect, there is no other BNE that features these properties. Moreover, as valuations become independent ( $\alpha$ converges to zero), the BNE $\beta$ converges to the classical truthful dominant strategy equilibrium in the Vickrey auction.

We show that perfect equilibrium is a useful tool to select the BNE $\beta$ in this auction. In particular, we show that $\beta$ is uniform-perfect. Next, we present a few classes of other BNEs for the auction. These BNEs are arguably less intuitive in the sense that types (at least partially) pool their bids. We show that perfection eliminates these equilibria, so that the BNE $\beta$ is uniquely selected by perfection from a large class of BNEs. Finally we argue that the usual standard selection criteria sincere bidding and dominant strategy equilibrium, have no bite in this context, and do not single out the BNE $\beta$. As the proofs in this section are technical in nature, we only discuss the main results, and defer the formal proofs to the appendix.

Consider the following sealed-bid second price auction $\Gamma^{\alpha}$ for a single indivisible object, where $\alpha$ is a parameter in $(0,1)$. There are two bidders, whose respective types $t_{1}$ and $t_{2}$ are drawn independently from $T_{1}=T_{2}=[0,1]$ according to the uniform distribution. The valuations of the bidders are symmetric and are given by $v_{1}\left(t_{1}, t_{2}\right)=5+t_{1}-\alpha t_{2}$ and $v_{2}\left(t_{1}, t_{2}\right)=5+t_{2}-\alpha t_{1}$. The set of available bids is $A_{1}=A_{2}=[5-\alpha, 6]$. The tie-breaking rule can be arbitrary, and plays no role in our analysis. ${ }^{13}$

\subsection{A symmetric BNE}

For each bidder $i$, define the pure strategy $B_{i}\left(t_{i}\right)=5+(1-\alpha) t_{i}$, and denote by $\beta_{i}$ the corresponding deterministic strategy. In this subsection we argue that, from a conceptual and normative point of view, the strategy pair $\beta=\left(\beta_{1}, \beta_{2}\right)$ is a natural candidate solution for the auction.

Concretely, we have the following claims. The strategy pair $\beta$ is a symmetric BNE, it separates the types, and it is continuous-even differentiable, so that bids increase smoothly with type. It is also unique in this, there is no other strategy pair that features all these properties. Moreover, as valuations become independent, so $\alpha \rightarrow 0$, the BNE $\beta$ converges to the classical truthful dominant strategy equilibrium in the Vickrey auction.

We first observe that $\beta$ is a BNE. The proof of this observation is deferred to Appendix II.

Proposition 24. The symmetric deterministic strategy profile $\beta=\left(\beta_{1}, \beta_{2}\right)$ is a BNE.

Next, we show that $\beta$ is the unique BNE within the class of differentiable separating pure strategy pairs. Let $\mathfrak{F}$ be the class of deterministic strategies in which the corresponding pure strategies $p_{i}: T_{i} \rightarrow A_{i}$ for bidders $i=1,2$ satisfy the following conditions:

(i) $p_{1}(0)=p_{2}(0)$ and $p_{1}(1)=p_{2}(1)$,

(ii) $p_{i}$ is continuous and strictly increasing in $t_{i}$.

Note that elements of $\mathfrak{F}$ separate the types of a player in the sense that the type can be inferred from the bid since no two types make the same bid. ${ }^{14}$ We have the following proposition.

Proposition 25. The BNE $\beta$ is the only pure BNE in class $\mathfrak{F}$.

Thus, the BNE $\beta$ is the unique continuous separating BNE. Moreover, as we already noted, when valuations become independent (so, when $\alpha \rightarrow 0$ ) $\beta$ converges to the truthful dominant strategy equilibrium in the Vickrey auction.

\subsection{The selective power of perfect BNE}

Thus, $\beta$ is a natural candidate as a solution for the auction we study. We show that perfect BNE does select the differentiable separating BNE $\beta$, from among many equilibria in the auction. We first show that $\beta$ is a perfect BNE.

Proposition 26. The BNE $\beta$ is uniform-perfect.

\footnotetext{
13 Bajoori (2011) and Liu (2014) study a similar class of auctions. In both papers valuations are increasing in both types, Liu (2014) studies common value auctions. Comparable to our results, they find that continuous BNEs are robust against perturbations, while discontinuous BNEs are not.

14 The first condition is mainly a normalization.
} 
We describe the idea of the proof. Let $C_{M}:[0,1] \rightarrow[5-\alpha, 6]$ be defined by $C_{M}(r)=5+(1-\alpha) r$ for all $r \in[0,1]$ and $\mu$ be the uniform distribution on [5- $\alpha, 6]$. The first idea would be to look at the sequences of completely mixed strategies $\hat{\beta}_{i}^{k}$ given by $\hat{\beta}_{i}^{k}\left(t_{i}, \cdot\right)=\left(1-\varepsilon_{k}\right) \delta_{C_{M}\left(t_{i}\right)}(\cdot)+\varepsilon_{k} \mu(\cdot)$ for both players $i=1,2$ and for every $t_{i}$, where $\delta$ is the Dirac measure and $\varepsilon_{k}$ is a sequence in $(0,1)$ converging to zero. One can verify that the sequence $\hat{\beta}_{i}^{k}$ satisfies condition (1) of Definition 21 , and $\lim _{k \rightarrow \infty} \rho^{w}\left(\hat{\beta}_{i}^{k}\left(t_{i}, \cdot\right), B R_{i}\left(t_{i}, \hat{\beta}^{k}\right)\right)=0$ for every $t_{i} \in(0,1)$. However, the latter does not hold for $t_{i}=0,1$. More precisely, one can easily check that $B R_{i}\left(0, \hat{\beta}^{k}\right)=\left\{5-\frac{1}{2} \alpha\right\}$ and $B R_{i}\left(1, \hat{\beta}^{k}\right)=\left\{6-\frac{1}{2} \alpha\right\}$, for every $k$. However, by defining the completely mixed strategies in a more delicate way, we can prove that $\left(\beta_{1}, \beta_{2}\right)$ is uniform-perfect. We introduce two more curves $C_{T}, C_{L}:[0,1] \rightarrow[5-\alpha, 6]$ by $C_{T}(r)=6-\alpha r^{2}$, and $C_{L}(r)=5-\alpha+\alpha(1-r)^{2}$. These curves are depicted in the picture below.

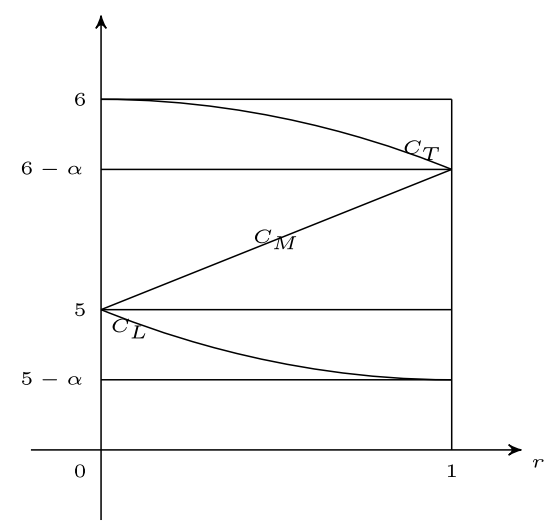

The curves $C_{T}$ and $C_{L}$ will be used to repair the above mentioned problem for types 0 and 1 . For every $k \in \mathbb{N}$, let $\varepsilon_{k}=\frac{1}{k+3}$ and $\beta_{1}^{k}: T_{1} \times \mathcal{A}_{1} \rightarrow[0,1]$ be the completely mixed strategy for bidder 1 which is given for every $t_{1} \in T_{1}=[0,1]$ by

$$
\beta_{1}^{k}\left(t_{1}, \cdot\right)=\left(1-2 \varepsilon_{k}-\varepsilon_{k}^{2}\right) \delta_{C_{M}\left(t_{1}\right)}(\cdot)+\varepsilon_{k} \delta_{C_{T}\left(t_{1}\right)}(\cdot)+\varepsilon_{k} \delta_{C_{L}\left(t_{1}\right)}(\cdot)+\varepsilon_{k}^{2} \mu(\cdot) .
$$

The cumulative probability distribution with respect to $\beta_{1}^{k}\left(t_{1}, \cdot\right)$ is shown on $A_{1}=[5-\alpha, 6]$ in the picture below.

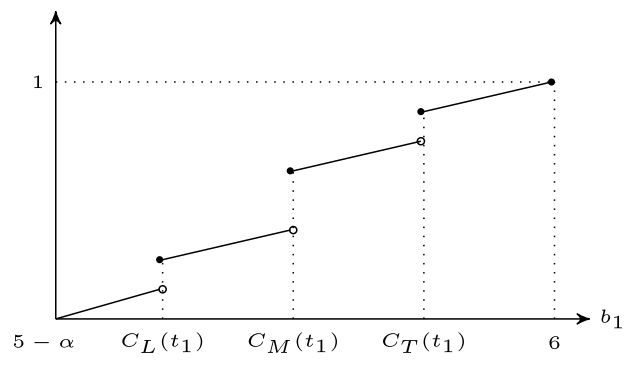

Similarly, define a completely mixed strategy $\beta_{2}^{k}$ for bidder 2. In Appendix II we will show that the sequence of strategy profiles $\left(\beta^{k}\right)_{k=1}^{\infty}=\left(\beta_{1}^{k}, \beta_{2}^{k}\right)_{k=1}^{\infty}$ satisfies conditions 1 and 2 of Definition 21 . Hence, the BNE $\beta$ is uniform-perfect.

Next, we present a few classes of other BNEs for the auction. They are arguably less intuitive in the sense that types (at least partially) pool their bids. We show that perfection eliminates these equilibria. We start with Wolf and Sheep BNEs.

Proposition 27. The deterministic strategy profile $\sigma=\left(\sigma_{1}, \sigma_{2}\right)$, given by $\sigma_{1}\left(t_{1}, \cdot\right)=\delta_{6}(\cdot)$ for every $t_{1} \in T_{1}$ and $\sigma_{2}\left(t_{2}, \cdot\right)=\delta_{5-\alpha}(\cdot)$ for every $t_{2} \in T_{2}$, is a BNE. However, $\sigma$ is not pointwise-perfect, and hence not uniform-perfect either.

It is worthwhile to note that the BNE introduced in Proposition 27 is just one of the many other BNEs of the same type. In all of this type of BNEs, the lowest bid of Wolf player is strictly larger than the highest bid of Sheep player.

The following proposition considers a deterministic BNE in which the corresponding pure strategies, as functions of the types, are not continuous. Also on this type of BNE we could construct many variations.

Proposition 28. The deterministic strategy profile $\eta=\left(\eta_{1}, \eta_{2}\right)$ is a BNE, where $\eta_{1}$ and $\eta_{2}$ correspond to the pure strategies $b_{1}$ and $b_{2}$ given by 


$$
b_{1}\left(t_{1}\right)=\left\{\begin{array}{ll}
5 & \text { if } t_{1} \in[0, x(\alpha)] \\
6 & \text { if } t_{1} \in(x(\alpha), 1]
\end{array} \quad \text { and } \quad b_{2}\left(t_{2}\right)= \begin{cases}5-\alpha & \text { if } t_{2} \in[0, y(\alpha)] \\
5.1 & \text { if } t_{2} \in(y(\alpha), 1]\end{cases}\right.
$$

with $x(\alpha)=\frac{0.4+2 \alpha}{4-\alpha^{2}}$ and $y(\alpha)=\frac{\alpha(0.2+\alpha)}{4-\alpha^{2}}$.

However, $\eta$ is not pointwise-perfect, and hence not uniform-perfect either.

Thus, perfection selects the equilibrium $\beta$ from among a large class of equilibria. Finally we argue that other standard selection criteria such as sincere bidding and dominance do not have a bite when types are interdependent (that is, when $\alpha>0) .{ }^{15}$ In particular, these selection criteria do not single out the separating BNE $\beta$ in this example.

Proposition 29. There does not exist a symmetric, deterministic, and strictly increasing BNE in which bidders bid sincerely given the opponent's bid function. In particular, the BNE $\beta$ is not sincere, in the sense that for each bidder $i$, there are types $t_{i}$ for which $B_{i}\left(t_{i}\right)$ does not equal his expected evaluation of the object given $t_{i}$.

Proposition 30. Neither bidder has a dominant strategy in the auction $\Gamma^{\alpha}$ in the following sense: neither bidder $i$ has a strategy $\beta_{i}$ such that for every type $t_{i}$, every strategy $\sigma_{i}$ of bidder $i$ and every strategy $\beta_{-i}$ of bidder $i$ 's opponents we have

$$
\mathbb{E}_{\beta_{-i}}\left(\Pi_{i} \mid t_{i}, \beta_{i}\right) \geq \mathbb{E}_{\beta_{-i}}\left(\Pi_{i} \mid t_{i}, \sigma_{i}\right)
$$

In fact, in Appendix II we prove a somewhat stronger statement, namely that in the above statement we could replace "every type $t_{i}$ " by "every type $t_{i}$ in a subset of types with a strictly positive measure".

Hence, the usual standard selection criteria sincere bidding and dominant strategy equilibrium, have no bite in the context of Bayesian games, and do not single out the BNE $\beta$.

\section{Appendix I: Proofs for the alternative notions of perfection in Section 4}

\subsection{Proof of Theorem 16}

Suppose that $\beta=\left(\beta_{1}, \ldots, \beta_{n}\right)$ is a pointwise-perfect strategy profile in such a Bayesian game. For $\beta$, take a sequence of strategy profiles $\left(\beta^{k}\right)_{k=1}^{\infty}$ as in Definition 15. Fix a player $i$ and a type $t_{i} \in T_{i}$. Due to condition (2) in Definition 15 , there exists a $K_{i}\left(t_{i}\right) \in \mathbb{N}$ such that $B R_{i}\left(t_{i}, \beta^{k}\right)$ is nonempty for every $k \geq K_{i}\left(t_{i}\right)$. Therefore, by condition $(2)$ once more, there exists a sequence $\left(\sigma_{i, t_{i}}^{k}\right)_{k=1}^{\infty}$ of probability measures on $\left(A_{i}, \mathcal{A}_{i}\right)$ such that $\sigma_{i, t_{i}}^{k} \in B R_{i}\left(t_{i}, \beta^{k}\right)$ for every $k \geq K_{i}\left(t_{i}\right)$ and $\rho^{w}\left(\beta_{i}^{k}\left(t_{i}, \cdot\right), \sigma_{i, t_{i}}^{k}\right) \rightarrow 0$ as $k \rightarrow \infty$. By condition (1) and by the triangle inequality for $\rho^{w}$, this implies that $\rho^{w}\left(\beta_{i}\left(t_{i}, \cdot\right), \sigma_{i, t_{i}}^{k}\right) \rightarrow 0$ as $k \rightarrow \infty$.

For every $k \geq K_{i}\left(t_{i}\right)$ and every probability measure $\sigma^{\prime}$ on $\left(A_{i}, \mathcal{A}_{i}\right)$, we have due to $\sigma_{i, t_{i}}^{k} \in B R_{i}\left(t_{i}, \beta^{k}\right)$ that $\mathbb{E}_{\beta_{-i}^{k}}\left(\Pi_{i} \mid\right.$ $\left.t_{i}, \sigma_{i}^{k}\right) \geq \mathbb{E}_{\beta_{-i}^{k}}\left(\Pi_{i} \mid t_{i}, \sigma^{\prime}\right)$, which means that

$$
\begin{aligned}
\int_{T_{-i}} \int_{A_{-i}} \int_{A_{i}} \Pi_{i}\left(\left(t_{i}, t_{-i}\right), a\right) \sigma_{i, t_{i}}^{k}\left(d a_{i}\right) \beta_{-i}^{k}\left(t_{-i}, d a_{-i}\right) \mu_{-i}\left(d t_{-i}\right) \\
\geq \int_{T_{-i}} \int_{A_{-i}} \int_{A_{i}} \Pi_{i}\left(\left(t_{i}, t_{-i}\right), a\right) \sigma^{\prime}\left(d a_{i}\right) \beta_{-i}^{k}\left(t_{-i}, d a_{-i}\right) \mu_{-i}\left(d t_{-i}\right) .
\end{aligned}
$$

According to Theorem 3.2 in Billingsley (1968), in a separable product space the weak convergence of the product measure is equivalent to the weak convergence of the marginal measures. Hence, because $A$ is separable, the weak convergence of $\sigma_{i, t_{i}}^{k}$ to $\beta_{i}\left(t_{i}, \cdot\right)$ and of $\beta_{j}^{k}\left(t_{j}, \cdot\right)$ to $\beta_{j}\left(t_{j}, \cdot\right)$ for every player $j \neq i$ and every type $t_{j} \in T_{j}$ implies that, for any $t_{-i} \in T_{-i}$, the product measure $\sigma_{i, t_{i}}^{k} \times\left(\times_{j \neq i} \beta_{j}^{k}\left(t_{j}, \cdot\right)\right)$ on $(A, \mathcal{A})$ weakly converges to the product measure $\beta_{i}\left(t_{i}, \cdot\right) \times\left(\times{ }_{j \neq i} \beta_{j}\left(t_{j}, \cdot\right)\right)$. Since the payoff function $\Pi_{i}\left(\left(t_{i}, t_{-i}\right), \cdot\right)$ is continuous on $A$ for every $t_{-i} \in T_{-i}$, by Fubini's theorem we have for every $t_{-i} \in T_{-i}$

$$
\begin{aligned}
\lim _{k \rightarrow \infty} \int_{A_{-i}} \int_{A_{i}} \Pi_{i} & \left(\left(t_{i}, t_{-i}\right), a\right) \sigma_{i, t_{i}}^{k}\left(d a_{i}\right) \beta_{-i}^{k}\left(t_{-i}, d a_{-i}\right) \\
& =\iint_{A_{-i}} \int_{A_{i}} \Pi_{i}\left(\left(t_{i}, t_{-i}\right), a\right) \beta_{i}\left(t_{i}, d a_{i}\right) \beta_{-i}\left(t_{-i}, d a_{-i}\right),
\end{aligned}
$$

$\overline{15}$ Given type $t_{i}$, bidder $i$ 's bid is sincere when he reports the expected value of the item, given the bid functions of the other bidders. 
and similarly

$$
\begin{aligned}
\lim _{k \rightarrow \infty} \int_{A_{-i}} \int_{A_{i}} \Pi_{i}\left(\left(t_{i}, t_{-i}\right), a\right) \sigma^{\prime}\left(d a_{i}\right) \beta_{-i}^{k}\left(t_{-i}, d a_{-i}\right) \\
\quad=\iint_{A_{-i}} \prod_{A_{i}} \Pi_{i}\left(\left(t_{i}, t_{-i}\right), a\right) \sigma^{\prime}\left(d a_{i}\right) \beta_{-i}\left(t_{-i}, d a_{-i}\right) .
\end{aligned}
$$

Thus, if we take the limit in inequality (2) when $k \rightarrow \infty$, by the dominated convergence theorem we obtain

$$
\begin{aligned}
\iint_{T_{-i}} \int_{A_{-i}} \Pi_{A_{i}} \Pi_{i}(t, a) & \beta_{i}\left(t_{i}, d a_{i}\right) \beta_{-i}\left(t_{-i}, d a_{-i}\right) \mu_{-i}\left(d t_{-i}\right) \\
& \geq \iint_{T_{-i}} \int_{A_{-i}} \int_{A_{i}} \Pi_{i}(t, a) \sigma^{\prime}\left(d a_{i}\right) \beta_{-i}\left(t_{-i}, d a_{-i}\right) \mu_{-i}\left(d t_{-i}\right),
\end{aligned}
$$

which means $\mathbb{E}_{\beta_{-i}}\left(\Pi_{i} \mid t_{i}, \beta_{i}\left(t_{i}, \cdot\right)\right) \geq \mathbb{E}_{\beta_{-i}}\left(\Pi_{i} \mid t_{i}, \sigma^{\prime}\right)$. Hence, $\beta_{i}\left(t_{i}, \cdot\right) \in B R_{i}\left(t_{i}, \beta\right)$. Since player $i$ and type $t_{i}$ were chosen arbitrary, the strategy profile $\beta$ is a BNE as claimed.

\subsection{Proof of Theorem 18}

For a perfect BNE $\beta$ as in Theorem 18, we can construct a desired pointwise-perfect BNE $\hat{\beta}$ as follows. For every player $i \in N$, every type $t_{i} \in S_{i}$, and every $k \in \mathbb{N}$, by assumption of the proposition, we can choose a probability measure $\sigma_{i, t_{i}}^{k} \in$ $B R_{i}\left(t_{i}, \beta^{k}\right)$. Now, define a profile of completely mixed strategies $\hat{\beta}^{k}$, for every $k \in \mathbb{N}$, by letting

$$
\hat{\beta}_{i}^{k}\left(t_{i}, \cdot\right)= \begin{cases}\beta_{i}^{k}\left(t_{i}, \cdot\right) & \text { if } t_{i} \in T_{i} \backslash S_{i} \\ \left(1-\frac{1}{k}\right) \sigma_{i, t_{i}}^{k}(\cdot)+\frac{1}{k} \beta_{i}^{k}\left(t_{i}, \cdot\right) & \text { if } t_{i} \in S_{i}\end{cases}
$$

for every player $i \in N$ and type $t_{i} \in T_{i}$.

In the remaining part of the proof, we use several fairly known results from analysis, which can all be found in Aliprantis and Border (1999). By assumption, $A_{i}$ is compact in the topology induced by the metric $d_{A_{i}}$, so the set $\Delta\left(A_{i}\right)$ of probability measures on $\left(A_{i}, \mathcal{A}_{i}\right)$ is also compact with respect to the topology induced by the weak metric. Consequently, the product space $\times_{i \in N} \times_{t_{i} \in S_{i}} \Delta\left(A_{i}\right)$ is compact in the product topology by Tychonoff's theorem, and because $S_{i}$ is countable, this topology is even metrizable. Therefore, this topological space is sequentially compact, which assures the existence of a subsequence $\left(k_{r}\right)_{r=1}^{\infty}$ so that $\sigma_{i, t_{i}}^{k_{r}}$ converges to some $\sigma_{i, t_{i}} \in \Delta\left(A_{i}\right)$, for every player $i \in N$ and every $t_{i} \in T_{i}$, with respect to the weak metric. So, define a strategy for every player $i \in N$ by

$$
\hat{\beta}_{i}\left(t_{i}, \cdot\right)= \begin{cases}\beta_{i}\left(t_{i}, \cdot\right) & \text { if } t_{i} \in T_{i} \backslash S_{i} \\ \sigma_{i, t_{i}}(\cdot) & \text { if } t_{i} \in S_{i}\end{cases}
$$

for every type $t_{i} \in T_{i}$. Notice that the profile $\hat{\beta}=\left(\hat{\beta}_{i}\right)_{i \in N}$ is a pointwise-perfect equilibrium. Indeed, for the sequence $\hat{\beta}^{k_{r}}$, condition (1) of Definition 15 is obviously satisfied, and so is condition (2) because $B R_{i}\left(t_{i}, \hat{\beta}^{k_{r}}\right)=B R_{i}\left(t_{i}, \beta^{k_{r}}\right)$ holds due to $\mu_{j}\left(S_{j}\right)=0$ for all players $j$.

\subsection{Proofs for Example 20}

A perfect BNE: First we construct a perfect BNE. Define $p_{1}\left(t_{1}\right)=U$ for every $t_{1} \in T_{1}, p_{2}\left(t_{2}\right)=a_{\infty}$ and $p_{3}\left(t_{3}\right)=b_{\infty}$. Let $\widetilde{\beta}_{i}$ be the deterministic strategy for each player $i$ corresponding to $p_{i}$. Now we prove that $\widetilde{\beta}=\left(\widetilde{\beta}_{1}, \widetilde{\beta}_{2}, \widetilde{\beta}_{3}\right)$ is a perfect BNE. Let $S_{1}=T_{1} \backslash\{(1,1, \ldots)\}$ and $S_{2}=S_{3}=\emptyset$. Further, take arbitrary completely mixed probability measures $\sigma_{2}$ and $\sigma_{3}$ on $\left(A_{2}, \mathcal{A}_{2}\right)$ and $\left(A_{3}, \mathcal{A}_{3}\right)$, respectively. For every $k \in \mathbb{N}$, define the completely mixed strategies

$$
\begin{aligned}
& \beta_{1}^{k}\left(t_{1}, \cdot\right)=\left(1-\frac{1}{k}\right) \cdot \delta_{U}(\cdot)+\frac{1}{k} \cdot \delta_{D}(\cdot) \text { for every } t_{1} \in T_{1} \\
& \beta_{2}^{k}\left(t_{2}, \cdot\right)=\left(1-\frac{1}{k^{2}}\right) \cdot \delta_{a_{k}}(\cdot)+\frac{1}{k^{2}} \cdot \sigma_{2}(\cdot) \\
& \beta_{3}^{k}\left(t_{3}, \cdot\right)=\left(1-\frac{1}{k^{2}}\right) \cdot \delta_{b_{k}}(\cdot)+\frac{1}{k^{2}} \cdot \sigma_{3}(\cdot)
\end{aligned}
$$

If player 2 plays actions $a_{k+1}$ against $\beta_{3}^{k}\left(t_{3}, \cdot\right)$, then he receives at least $\left(1-\frac{1}{k^{2}}\right) \frac{1}{k+1}$, whereas he receives at most $\frac{1}{k+2}$ by playing an action $a_{\ell}$ with $\ell>k+1$ and receives at most $\frac{1}{2 k^{2}}$ by playing an action $a_{\ell}$ with $\ell<k+1$. Thus, for every $k \geq 3$, action $a_{k+1}$ is player 2's (unique) best response to $\beta_{3}^{k}\left(t_{3}, \cdot\right)$, and for similar reasons, action $b_{k+1}$ is player 3's (unique) best 
response to $\beta_{2}^{k}\left(t_{2}, \cdot\right)$. Based on this observation, one can check easily that $S_{1}, S_{2}, S_{3}$ and the sequence $\beta^{k}=\left(\beta_{1}^{k}, \beta_{2}^{k}, \beta_{3}^{k}\right)$ satisfy the conditions of Definition 7 for $\widetilde{\beta}$. Thus, $\widetilde{\beta}$ is a perfect BNE indeed.

No pointwise-perfect strategy profile: Now we claim that there is no pointwise-perfect strategy profile in this game. (Note that $S_{1}$ above is uncountable, so Proposition 18 does not apply.) Suppose by way of contradiction that $\beta=\left(\beta_{1}, \beta_{2}, \beta_{3}\right)$ is a pointwise-perfect strategy profile, with some sequence $\left(\beta^{k}\right)_{k=1}^{\infty}$ as required in Definition 15.

First we prove that $\beta_{2}\left(t_{2}, \cdot\right)$ and $\beta_{3}\left(t_{3}, \cdot\right)$ put probability 1 on action $a_{\infty}$ and $b_{\infty}$, respectively, i.e. $\beta_{2}\left(t_{2},\left\{a_{\infty}\right\}\right)=$ $\beta_{3}\left(t_{3},\left\{b_{\infty}\right\}\right)=1$. Suppose by way of contradiction that $\beta_{2}\left(t_{2},\left\{a_{\infty}\right\}\right)<1$; the proof is similar if $\beta_{3}\left(t_{3},\left\{b_{\infty}\right\}\right)<1$. Let $m^{*}=\min \left\{m \in \mathbb{N} \mid \beta_{2}\left(t_{2},\left\{a_{m}\right\}\right)>0\right\}$. Since player 3 can get a positive payoff against $\beta_{2}\left(t_{2}, \cdot\right)$, and $\beta_{3}\left(t_{3}, \cdot\right)$ is a best response to $\beta_{2}\left(t_{2}, \cdot\right)$, we must have $\beta_{3}\left(t_{3},\left\{b_{\infty}\right\}\right)=0$. So, also player 2 can get a positive payoff against $\beta_{3}\left(t_{3}, \cdot\right)$. Let $\ell^{*}=\min \left\{\ell \in \mathbb{N} \mid \beta_{3}\left(t_{3},\left\{b_{\ell}\right\}\right)>0\right\}$. Now, if $m^{*} \leq \ell^{*}$, then action $a_{m^{*}}$ gives payoff zero to player 2 , which is a contradiction as $\beta_{2}\left(t_{2},\left\{a_{m^{*}}\right\}\right)>0$. Similarly, if $\ell^{*} \leq m^{*}$, then action $b_{\ell^{*}}$ gives payoff zero to player 3 , which is a contradiction as $\beta_{3}\left(t_{3},\left\{b_{\ell^{*}}\right\}\right)>0$. So, $\beta_{2}\left(t_{2},\left\{a_{\infty}\right\}\right)=\beta_{3}\left(t_{3},\left\{b_{\infty}\right\}\right)=1$ must hold indeed.

For every $k \in \mathbb{N}$, let $\sigma^{k}$ be the probability measure on $\left(A_{2}, \mathcal{A}_{2}\right)$ defined by $\sigma^{k}\left(a_{\infty}\right)=0$ and

$$
\sigma^{k}\left(a_{m}\right)=\frac{\beta_{2}^{k}\left(t_{2},\left\{a_{m}\right\}\right)}{1-\beta_{2}^{k}\left(t_{2},\left\{a_{\infty}\right\}\right)}
$$

for every $m \in \mathbb{N}$. So, $\sigma^{k}\left(a_{m}\right)$ equals the probability that action $a_{m}$ is chosen with respect to $\beta_{2}^{k}\left(t_{2}, \cdot\right)$ conditioned on the event that $a_{\infty}$ is not chosen. We now claim that for every $m \in \mathbb{N}$

$$
\lim _{k \rightarrow \infty} \sigma^{k}\left(\left\{a_{1}, \ldots, a_{m}\right\}\right)=0 .
$$

Suppose by way of contradiction that for some $m \in \mathbb{N}$ there exists a $Z>0$ and a subsequence $\left(k_{r}\right)_{r=1}^{\infty}$ such that $\sigma^{k_{r}}\left(\left\{a_{1}, \ldots, a_{m}\right\}\right) \geq Z$ for every $r \in \mathbb{N}$. Notice that, for every $r \in \mathbb{N}$, action $b_{m+1}$ gives in expectation at least $\frac{Z}{m+1}$ to player 3 against $\sigma^{k_{r}}$, hence at least $\left(1-\beta_{2}^{k_{r}}\left(t_{2},\left\{a_{\infty}\right\}\right)\right) \cdot \frac{Z}{m+1}$ against $\beta^{k_{r}}$. Now consider any action $b_{w}$ with $w>\frac{m+1}{Z}$. Since player 3's highest payoff for action $b_{w}$ is $\frac{1}{w}$, action $b_{w}$ gives player 3 in expectation strictly less than $\frac{Z}{m+1}$ against $\sigma^{k_{r}}$, hence strictly less than $\left(1-\beta_{2}^{k_{r}}\left(t_{2},\left\{a_{\infty}\right\}\right)\right) \cdot \frac{Z}{m+1}$ against $\beta^{k_{r}}$. Therefore, player 3's best responses are in $\left\{b_{1}, \ldots, b_{\left\lfloor\frac{m+1}{Z}\right\rfloor}\right\}$ against $\beta^{k_{r}}$ for any $r \in \mathbb{N}$. Since player 3's best responses along this subsequence are not approaching $b_{\infty}$ with respect to the weak metric, the sequence $\beta^{k}$ cannot satisfy both conditions (1) and (2) of Definition 15. Thus, (3) holds as claimed.

Let $k_{1}=1$ and $m_{1} \in \mathbb{N}$ be such that $\sigma^{k_{1}}\left(\left\{a_{1}, \ldots, a_{m_{1}}\right\}\right)>\frac{1}{2}$. Then, by (3), there exists a $k_{2}>k_{1}$ and an $m_{2}>m_{1}$ such that $\sigma^{k_{2}}\left(\left\{a_{m_{1}+1}, \ldots, a_{m_{2}}\right\}\right)>\frac{1}{2}$. By repeating this argument, we obtain in $\mathbb{N}$ two strictly increasing sequences $\left(m_{r}\right)_{r=1}^{\infty}$ and $\left(k_{r}\right)_{r=1}^{\infty}$ such that for every $r \in \mathbb{N}$

$$
\sigma^{k_{r}}\left(\left\{a_{m} \mid m \in W_{r}\right\}\right)>\frac{1}{2},
$$

where $W_{r}=\left\{m_{r-1}+1, \ldots, m_{r}\right\}$ and $m_{0}=0$. Note that the sets $W_{r}, r \in \mathbb{N}$, form a partition of $\mathbb{N}$.

Now let $t_{1}$ be the type in $\{0,1\}^{\mathbb{N}}$ such that $f_{m}\left(t_{1}\right)=1$ if $m \in W_{r}$ for an odd $r \in \mathbb{N}$ and $f_{m}\left(t_{1}\right)=0$ if $m \in W_{r}$ for an even $r \in \mathbb{N}$. Take an arbitrary odd $r \in \mathbb{N}$. Then, $f_{m}\left(t_{1}\right)=1$ for any $m \in W_{r}$, and since $\sigma^{k_{r}}\left(\left\{a_{m} \mid m \in W_{r}\right\}\right)>\frac{1}{2}$ due to (4), player 1's unique best response to $\beta^{k_{r}}$ is action $U$. Similarly, when $r \in \mathbb{N}$ is even, player 1's unique best response to $\beta^{k_{r}}$ is action $D$. So, the sequence $\beta^{k}$ cannot satisfy both conditions (1) and (2) of Definition 15 for type $t_{1}$, which is a contradiction. Consequently, there is no pointwise-perfect strategy profile in this game.

\section{Appendix II: Proofs for the auction in Section 5}

Throughout the Appendix II, we will use the notion $\mathbb{E}_{\tau_{1}}\left(\Pi_{2} \mid t_{2}, b_{2}\right)$ for bidder 2's expected profit given his type $t_{2}$ and bid $b_{2}$ against a strategy $\tau_{1}$ of bidder 1 . If bidder 1 makes a bid $b_{1}>b_{2}$, then bidder 2 does not win the object and has zero profit. Therefore, if bidding $b_{2}$ against $\tau_{1}$ leads to a tie with zero ex-ante probability, then we have

$$
\mathbb{E}_{\tau_{1}}\left(\Pi_{2} \mid t_{2}, b_{2}\right)=\int_{0}^{1} \int_{5-\alpha}^{b_{2}}\left(5+t_{2}-\alpha t_{1}-b_{1}\right) \tau_{1}\left(t_{1}, d b_{1}\right) d t_{1} .
$$

Since the function $5+t_{2}-\alpha t_{1}-b_{1}$ is bounded on $[0,1] \times[5-\alpha, 6]$ and measurable with respect to $\mathcal{T} \times \mathcal{A}$, then according to the Theorem 10.2.1 in Dudley (2002), $\mathbb{E}_{\tau_{1}}\left(\Pi_{2} \mid t_{2}, b_{2}\right)$ is well-defined.

In the special case when $\tau_{1}$ is a deterministic strategy, i.e. when $\tau_{1}\left(t_{1}, \cdot\right)=\delta_{p_{1}\left(t_{1}\right)}(\cdot)$ for a pure strategy $p_{1}$, we have

$$
\int_{5-\alpha}^{b_{2}}\left(5+t_{2}-\alpha t_{1}-b_{1}\right) \tau_{1}\left(t_{1}, d b_{1}\right)= \begin{cases}5+t_{2}-\alpha t_{1}-p_{1}\left(t_{1}\right) & \text { if } p_{1}\left(t_{1}\right) \in\left[5-\alpha, b_{2}\right] \\ 0 & \text { if } p_{1}\left(t_{1}\right) \in\left(b_{2}, 6\right] .\end{cases}
$$


Hence, if $p_{1}$ is increasing and $b_{2} \in p_{1}\left(T_{1}\right)$, then we have

$$
\mathbb{E}_{\tau_{1}}\left(\Pi_{2} \mid t_{2}, b_{2}\right)=\int_{0}^{p_{1}^{-1}\left(b_{2}\right)}\left(5+t_{2}-\alpha t_{1}-p_{1}\left(t_{1}\right)\right) d t_{1}
$$

\subsection{Proof of Proposition 24}

We prove that the deterministic strategy profile $\beta=\left(\beta_{1}, \beta_{2}\right)$ in which $\beta_{i}$ corresponds to the pure strategy $B_{i}\left(t_{i}\right)=$ $5+(1-\alpha) t_{i}$ for each bidder $i$, is a BNE. For this purpose, we prove that $\beta_{2}$ is a best response against $\beta_{1}$. Then, due to symmetry, $\beta_{1}$ is also a best response against $\beta_{2}$, and the proof will be complete.

According to (6), if $b_{2} \in[5,6-\alpha]$ then we have

$$
\begin{aligned}
\mathbb{E}_{\beta_{1}}\left(\Pi_{2} \mid t_{2}, b_{2}\right) & =\int_{0}^{B_{1}^{-1}\left(b_{2}\right)}\left(5+t_{2}-\alpha t_{1}-B_{1}\left(t_{1}\right)\right) d t_{1} \\
& =\int_{0}^{\frac{b_{2}-5}{1-\alpha}}\left(5+t_{2}-\alpha t_{1}-5-(1-\alpha) t_{1}\right) d t_{1} \\
& =\int_{0}^{\frac{b_{2}-5}{1-\alpha}}\left(t_{2}-t_{1}\right) d t_{1} \\
& =t_{2}\left(\frac{b_{2}-5}{1-\alpha}\right)-\frac{1}{2}\left(\frac{b_{2}-5}{1-\alpha}\right)^{2} .
\end{aligned}
$$

By taking the first derivative with respect to $b_{2}$

$$
\frac{d}{d b_{2}} \mathbb{E}_{\beta_{1}}\left(\Pi_{2} \mid t_{2}, b_{2}\right)=\frac{t_{2}}{1-\alpha}-\frac{1}{1-\alpha}\left(\frac{b_{2}-5}{1-\alpha}\right) .
$$

It is clear that

$$
\frac{d}{d b_{2}} \mathbb{E}_{\beta_{1}}\left(\Pi_{2} \mid t_{2}, 5\right) \geq 0 \text { and } \frac{d}{d b_{2}} \mathbb{E}_{\beta_{1}}\left(\Pi_{2} \mid t_{2}, 6-\alpha\right) \leq 0,
$$

which implies that the maximum of $\mathbb{E}_{\beta_{1}}\left(\Pi_{2} \mid t_{2}, b_{2}\right)$ over $b_{2} \in[5,6-\alpha]$ is attained where $\frac{d}{d b_{2}} \mathbb{E}_{\beta_{1}}\left(\Pi_{2} \mid t_{2}, b_{2}\right)=0$. This happens exactly when

$$
b_{2}=5+(1-\alpha) t_{2}=B_{2}\left(t_{2}\right) .
$$

Notice that, for bidder 2, bidding less than 5 is never better than bidding 5 exactly, because all bids less than or equal to 5 win against $\beta_{1}$ with probability 0 . Similarly, bidding more than $6-\alpha$ is never better than bidding $6-\alpha$ exactly, because all bids larger than or equal to $6-\alpha$ win against $\beta_{1}$ with probability 1 . Therefore, $\beta_{2}$ is a best response to $\beta_{1}$ as claimed.

\subsection{Proof of Proposition 25}

Consider two pure strategies $p_{1}$ and $p_{2}$ that satisfy conditions (i), (ii) of Proposition 25, and the corresponding deterministic strategies $\tau_{1}$ and $\tau_{2}$. Suppose that $\left(\tau_{1}, \tau_{2}\right)$ is a BNE. Our goal is to show that $p_{1}\left(t_{1}\right)=5+(1-\alpha) t_{1}$ and $p_{2}\left(t_{2}\right)=5+(1-\alpha) t_{2}$.

Recall that $T_{1}=T_{2}=[0,1]$, and $A_{1}=A_{2}=[5-\alpha, 6]$. Notice that $p_{1}: T_{1} \rightarrow A_{1}$ and $p_{2}: T_{2} \rightarrow A_{2}$ are continuous and invertible, and therefore they have a continuous inverse. Thus, because $p_{1}\left(T_{1}\right)=p_{2}\left(T_{2}\right)$, the function $\hat{p}=p_{1}^{-1} \circ p_{2}$ is well defined and it is a continuous strictly increasing bijection from $T_{2}$ to $T_{1}$.

First we argue that $p_{2}(0) \leq 5$. Suppose by way of contradiction that $p_{2}(0)>5$. According to (6) we have

$$
\mathbb{E}_{\tau_{1}}\left(\Pi_{2} \mid t_{2}, p_{2}\left(t_{2}\right)\right)=\int_{0}^{\hat{p}\left(t_{2}\right)}\left(5+t_{2}-\alpha t_{1}-p_{1}\left(t_{1}\right)\right) d t_{1} .
$$

Notice that if $t_{2}>0$ then $\hat{p}\left(t_{2}\right)>0$ due to conditions (i) and (ii), and moreover, for every type $t_{2}<p_{2}(0)-5$ we have 


$$
5+t_{2}-\alpha t_{1}-p_{1}\left(t_{1}\right) \leq 5+t_{2}-p_{1}\left(t_{1}\right) \leq 5+t_{2}-p_{1}(0)=5+t_{2}-p_{2}(0)<0
$$

Therefore, $\mathbb{E}_{\tau_{1}}\left(\Pi_{2} \mid t_{2}, p_{2}\left(t_{2}\right)\right)$ is strictly negative. However, as $p_{1}(0)=p_{2}(0)>5$, bidder 2 can get zero by bidding 5 for instance, which never wins. This is a contradiction with the assumption that $\tau_{2}$ is a best response to $\tau_{1}$, so $p_{2}(0) \leq 5$ holds indeed.

Now we prove that $p_{2}(1) \geq 6-\alpha$. Write $c:=6-\alpha-p_{2}(1)$ and suppose by way of contradiction that $c>0$. According to (6), if we compare bids $p_{2}(1)$ and $p_{2}\left(t_{2}\right)$ for bidder 2 when his type is $t_{2}$, we obtain

$$
\mathbb{E}_{\tau_{1}}\left(\Pi_{2} \mid t_{2}, p_{2}(1)\right)-\mathbb{E}_{\tau_{1}}\left(\Pi_{2} \mid t_{2}, p_{2}\left(t_{2}\right)\right)=\int_{\hat{p}\left(t_{2}\right)}^{1}\left(5+t_{2}-\alpha t_{1}-p_{1}\left(t_{1}\right)\right) d t_{1} .
$$

Notice that if $t_{2} \in(1-c, 1)$, then $\hat{p}\left(t_{2}\right)<1$ due to conditions (i) and (ii), and moreover, for every $t_{1} \in[0,1]$ we have

$$
\begin{aligned}
5+t_{2}-\alpha t_{1}-p_{1}\left(t_{1}\right) & \geq 5+t_{2}-\alpha-p_{1}(1) \\
& =5+t_{2}-\alpha-p_{2}(1) \\
& =5+t_{2}-\alpha+c-6+\alpha \\
& =-1+t_{2}+c \\
& >0 .
\end{aligned}
$$

Therefore, for every $t_{2} \in(1-c, 1)$

$$
\mathbb{E}_{\tau_{1}}\left(\Pi_{2} \mid t_{2}, p_{2}(1)\right)-\mathbb{E}_{\tau_{1}}\left(\Pi_{2} \mid t_{2}, p_{2}\left(t_{2}\right)\right)>0
$$

which is in contradiction with the assumption that $\tau_{2}$ is a best response to $\tau_{1}$. Hence, $p_{2}(1) \geq 6-\alpha$ holds indeed.

Now, we calculate bidder 2's best response bids against $\tau_{1}$, given his type $t_{2}$. It is clear that, for any type $t_{2}$, bidding $p_{2}(0)$ is not worse than any bid in $\left[5-\alpha, p_{2}(0)\right]$, because all these bids win with probability zero against $\tau_{1}$. Similarly, bidding $p_{2}(1)$ is not worse than any bid in $\left[p_{2}(1), 6\right]$, because all these bids win with probability 1 against $\tau_{1}$. It remains to determine the best bids in the interval $\left[p_{2}(0), p_{2}(1)\right]$. By (6), we have for every bid $b_{2} \in\left[p_{2}(0), p_{2}(1)\right]$ that

$$
\begin{aligned}
\mathbb{E}_{\tau_{1}}\left(\Pi_{2} \mid t_{2}, b_{2}\right) & =\int_{0}^{p_{1}^{-1}\left(b_{2}\right)}\left(5+t_{2}-\alpha t_{1}-p_{1}\left(t_{1}\right)\right) d t_{1} \\
& =\left[\left(5+t_{2}\right) t_{1}-\frac{\alpha}{2} t_{1}^{2}\right]_{0}^{p_{1}^{-1}\left(b_{2}\right)}-\int_{0}^{p_{1}^{-1}\left(b_{2}\right)} p_{1}\left(t_{1}\right) d t_{1} \\
& =\left(5+t_{2}\right) p_{1}^{-1}\left(b_{2}\right)-\frac{\alpha}{2}\left(p_{1}^{-1}\left(b_{2}\right)\right)^{2}-\int_{0}^{p_{1}^{-1}\left(b_{2}\right)} p_{1}\left(t_{1}\right) d t_{1} .
\end{aligned}
$$

Bidder 2 wants to maximize this expression over $b_{2} \in\left[p_{2}(0), p_{2}(1)\right]=\left[p_{1}(0), p_{1}(1)\right]$. So, using the substitution $y=p_{1}^{-1}\left(b_{2}\right)$ the optimization problem is equivalent to maximizing over $y \in[0,1]$ the function

$$
F(y)=\left(5+t_{2}\right) \cdot y-\frac{\alpha}{2} \cdot y^{2}-\int_{0}^{y} p_{1}\left(t_{1}\right) d t_{1} .
$$

Since $p_{1}$ is continuous, $F$ is continuously differentiable. So, the first order condition yields

$$
5+t_{2}-\alpha \cdot y-p_{1}(y)=0 .
$$

Note that by assumption the type $t_{2}$ for which $p_{2}\left(t_{2}\right)=b_{2}$ solves this condition, and that $y=p_{1}^{-1}\left(b_{2}\right)$ by definition. The first order condition can be rewritten to

$$
p_{1}(y)=5+t_{2}-\alpha \cdot y .
$$

Substituting $y=p_{1}^{-1}\left(b_{2}\right)$ yields

$$
b_{2}=5+t_{2}-\alpha \cdot p_{1}^{-1}\left(b_{2}\right) .
$$


Next, substituting $p_{2}\left(t_{2}\right)=b_{2}$ yields

$$
p_{2}\left(t_{2}\right)=5+t_{2}-\alpha \cdot p_{1}^{-1}\left(p_{2}\left(t_{2}\right)\right) .
$$

In the same way we find

$$
p_{1}\left(t_{1}\right)=5+t_{1}-\alpha \cdot p_{2}^{-1}\left(p_{1}\left(t_{1}\right)\right) .
$$

Now take any $z \in[0,1]$. We argue that $p_{1}(z)=p_{2}(z)$. If $\alpha=0$, this is immediately clear. Suppose $\alpha>0$. Assume without loss that $p_{1}(z)>p_{2}(z)$. We derive a contradiction.

Since $p_{1}(z)>p_{2}(z)$, we have $z>p_{1}^{-1}\left(p_{2}(z)\right)$ and also $p_{2}^{-1}\left(p_{1}(z)\right)>z$. So, $p_{2}^{-1}\left(p_{1}(z)\right)>z>p_{1}^{-1}\left(p_{2}(z)\right)$. Taking $t_{1}=$ $z=t_{2}$, we find

$$
p_{1}(z)-p_{2}(z)=\alpha \cdot\left[p_{1}^{-1}\left(p_{2}(z)\right)-p_{2}^{-1}\left(p_{1}(z)\right)\right]<0 .
$$

This contradicts the assumption that $p_{1}(z)>p_{2}(z)$.

Thus, $p_{1}(z)=p_{2}(z)$ for all $z \in[0,1]$, so that $p_{2}^{-1}\left(p_{1}\left(t_{1}\right)\right)=t_{1}$ and $p_{1}^{-1}\left(p_{2}\left(t_{2}\right)\right)=t_{2}$. It follows that $p_{1}\left(t_{1}\right)=5+(1-\alpha) t_{1}$ and $p_{2}\left(t_{2}\right)=5+(1-\alpha) t_{2}$. This completes the proof of Proposition 25 .

\subsection{Proof of Proposition 29}

Suppose that $\left(b_{1}, b_{2}\right)$ is symmetric and deterministic, $b_{1}=b_{2}$ is strictly increasing, and $b_{1}$ is sincere given $b_{2}$. Since $b_{1}=b_{2}$ by symmetry, and since $b_{1}=b_{2}$ is strictly increasing, we have that

$$
\mathbb{E}\left(t_{2} \mid b_{1}\left(t_{1}\right)>b_{2}\left(t_{2}\right)\right)=\mathbb{E}\left(t_{2} \mid t_{1}>t_{2}\right)=\frac{1}{2} t_{1} .
$$

So, since $b_{1}$ is sincere given $b_{2}$, using the above equality we have that

$$
b_{1}\left(t_{1}\right)=\mathbb{E}\left(v_{1}\left(t_{1}, t_{2}\right) \mid b_{1}\left(t_{1}\right)>b_{2}\left(t_{2}\right)\right)=\mathbb{E}\left(5+t_{1}-\alpha t_{2} \mid b_{1}\left(t_{1}\right)>b_{2}\left(t_{2}\right)\right)=5+t_{1}-\frac{\alpha}{2} t_{1} .
$$

So, $b_{1}\left(t_{1}\right)=5+t_{1}-\frac{\alpha}{2} t_{1}$ and $b_{2}\left(t_{2}\right)=5+t_{2}-\frac{\alpha}{2} t_{2}$. This implies that $\left(b_{1}, b_{2}\right) \in \mathfrak{F}$. So, since $b_{1}\left(t_{1}\right)>B_{1}\left(t_{1}\right)$ for every type $t_{1}>0$, Proposition 25 shows that $\left(b_{1}, b_{2}\right)$ does not constitute a BNE.

It is worthwhile to mention that even if we look at the situation in which bidder 1 naively bids his ex ante expected valuation, the same conclusion holds. In that case bidder 1's expected evaluation of the object given his type $t_{1}$ is

$$
\mathbb{E}\left(v_{1}\left(t_{1}, t_{2}\right) \mid t_{1}\right)=\mathbb{E}\left(5+t_{1}-\alpha t_{2} \mid t_{1}\right)=5+t_{1}-\frac{\alpha}{2},
$$

and the same reasoning as before shows that also this does not yield a BNE.

\subsection{Proof of Proposition 30}

We prove that neither bidder has a dominant strategy. To this end we prove that every strategy $\sigma_{i}$ of player $i=1,2$ is not dominant. Suppose $\sigma_{2}$ is a dominant strategy for bidder 2. Notice that if bidder 1 chooses strategy $\beta_{1}$ and if bidder 2 is given type $t_{2} \in(0,1)$, then bidding $\beta_{2}$ is bidder 2's unique best response (see the proof of Proposition 24). This implies that $\sigma_{2}=\beta_{2}$. Now we show that the strategies $\beta_{2}$ is not in dominant strategies. To this end we show that $\beta_{2}$ is not a best response against all strategies of bidder 1 . Suppose that bidder 1's strategy is given by $\lambda_{1}\left(t_{1}, \cdot\right)=\delta_{b_{1}\left(t_{1}\right)}(\cdot)$ for every $t_{1} \in T_{1}$, where

$$
b_{1}\left(t_{1}\right)= \begin{cases}\frac{11}{2} & \text { if } t_{1} \in\left[0, \frac{1}{2}\right] \\ 6 & \text { if } t_{1} \in\left(\frac{1}{2}, 1\right] .\end{cases}
$$

We prove that if $t_{2}=\frac{1+\alpha}{2}$, then $\beta_{2}$ is not a best response for bidder 2 against $\lambda_{1}$. Notice that bidder 2 gets zero by bidding $B_{2}\left(\frac{1+\alpha}{2}\right)$, because it never wins against $\lambda_{1}$ due to $B_{2}\left(\frac{1+\alpha}{2}\right)=5+(1-\alpha)\left(\frac{1+\alpha}{2}\right)<\frac{11}{2}$. However, by (6), bidder 2's expected profit given type $t_{2}=\frac{1+\alpha}{2}$ and $b_{2} \in\left(\frac{11}{2}, 6\right)$ is

$$
\mathbb{E}_{\lambda_{1}}\left(\Pi_{2} \mid \frac{1+\alpha}{2}, b_{2}\right)=\int_{0}^{\frac{1}{2}}\left(5+\frac{1+\alpha}{2}-\alpha t_{1}-\frac{11}{2}\right) d t_{1}=\frac{\alpha}{8}>0 .
$$

This shows that if $t_{2}=\frac{1+\alpha}{2}$ then bidding $b_{2} \in\left(\frac{11}{2}, 6\right)$ is strictly better than bidding $B_{2}\left(\frac{1+\alpha}{2}\right)$. Hence, $\beta_{2}$ is not in dominant strategies. This proves that bidder 2 does not have a dominant strategy at all, and by symmetry the same is true for bidder 1 . The proof is complete. 


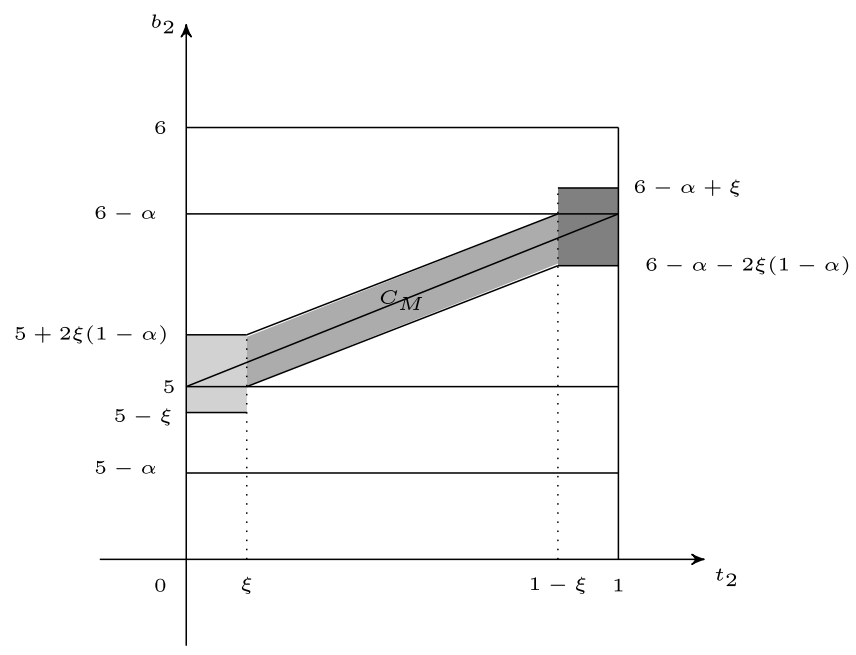

Fig. 1. Bidder 2's best response bids.

\subsection{Proof of Proposition 26} by

We prove that the BNE $\beta=\left(\beta_{1}, \beta_{2}\right)$ is uniform-perfect. For this purpose, define the curves $C_{M}, C_{T}, C_{L}:[0,1] \rightarrow[5-\alpha, 6]$

$$
\begin{aligned}
& C_{M}\left(t_{1}\right)=5+(1-\alpha) t_{1}, \\
& C_{T}\left(t_{1}\right)=6-\alpha t_{1}^{2}, \\
& C_{L}\left(t_{1}\right)=5-\alpha+\alpha\left(1-t_{1}\right)^{2} .
\end{aligned}
$$

Let $\mu$ be the uniform distribution on $A_{1}=[5-\alpha, 6]$. For every $k \in \mathbb{N}$, let $\varepsilon_{k}=\frac{1}{k+3}$ and $\beta_{1}^{k}: T_{1} \times \mathcal{A}_{1} \rightarrow[0,1]$ be the completely mixed strategy for bidder 1 which is given for every $t_{1} \in T_{1}=[0,1]$ by

$$
\beta_{1}^{k}\left(t_{1}, \cdot\right)=\left(1-2 \varepsilon_{k}-\varepsilon_{k}^{2}\right) \delta_{C_{M}\left(t_{1}\right)}(\cdot)+\varepsilon_{k} \delta_{C_{T}\left(t_{1}\right)}(\cdot)+\varepsilon_{k} \delta_{C_{L}\left(t_{1}\right)}(\cdot)+\varepsilon_{k}^{2} \mu(\cdot) .
$$

Similarly, define a completely mixed strategy $\beta_{2}^{k}$ for bidder 2 . We show that the sequence of strategy profiles $\left(\beta^{k}\right)_{k=1}^{\infty}=$ $\left(\beta_{1}^{k}, \beta_{2}^{k}\right)_{k=1}^{\infty}$ satisfies conditions 1 and 2 of Definition 21.

It is clear from the definition of $\beta_{i}\left(t_{i}, \cdot\right)$ and $\beta_{i}^{k}\left(t_{i}, \cdot\right)$, for each bidder $i$, that

$$
\sup _{t_{i} \in T_{i}} \rho^{w}\left(\beta_{i}^{k}\left(t_{i}, \cdot\right), \beta_{i}\left(t_{i}, \cdot\right)\right)=2 \varepsilon_{k}+\varepsilon_{k}^{2} .
$$

Hence, condition 1 of Definition 21 is satisfied. Condition 2 of Definition 21 follows from the claim below.

Claim. For every $\xi \in(0, \alpha)$, there is a $K_{\xi} \in \mathbb{N}$ such that for every $k>K_{\xi}$ we have:

1. For every $t_{2} \in[0, \xi)$, the maximum of $\mathbb{E}_{\beta_{1}^{k}}\left(\Pi_{2} \mid t_{2}, b_{2}\right)$ over $b_{2} \in[5-\alpha, 6]$ can only be attained within $b_{2} \in[5-\xi, 5+2 \xi(1-\alpha)]$.

2. For every $t_{2} \in[\xi, 1-\xi]$, the maximum of $\mathbb{E}_{\beta_{1}^{k}}\left(\Pi_{2} \mid t_{2}, b_{2}\right)$ over $b_{2} \in[5-\alpha, 6]$ can only be attained within $b_{2} \in\left[C_{M}\left(t_{2}\right)-\xi(1-\right.$ $\left.\alpha), C_{M}\left(t_{2}\right)+\xi(1-\alpha)\right]$.

3. For every $t_{2} \in(1-\xi, 1]$ the maximum of $\mathbb{E}_{\beta_{1}^{k}}\left(\Pi_{2} \mid t_{2}, b_{2}\right)$ over $b_{2} \in[5-\alpha, 6]$ can only be attained within $b_{2} \in[6-\alpha-2 \xi(1-$ $\alpha), 6-\alpha+\xi]$.

The above claim is illustrated in Fig. 1. According to the claim, the gray area includes all best response bids for bidder 2 if $k>K_{\xi}$.

Take an arbitrary $\xi \in\left(0, \min \left\{\alpha, \frac{1}{2}\right\}\right)$. To prove this claim, it is enough to show that the following Claims 1,2 and 3 are valid. These claims corresponds to three cases, i.e., $6-\alpha \leq b_{2} \leq 6,5-\alpha \leq b_{2} \leq 5$ and $5 \leq b_{2} \leq 6-\alpha$.

Claim 1 (for the case $6-\alpha \leq b_{2} \leq 6$ ). There is an $M_{\xi}^{1} \in \mathbb{N}$ such that for every $k>M_{\xi}^{1}$ we have:

1. For every $t_{2} \in[0,1-\xi]$, the maximum of $\mathbb{E}_{\beta_{1}^{k}}\left(\Pi_{2} \mid t_{2}, b_{2}\right)$ over $b_{2} \in[6-\alpha, 6]$ is attained at $b_{2}=6-\alpha$.

2. For every $t_{2} \in(1-\xi, 1]$, the maximum of $\mathbb{E}_{\beta_{1}^{k}}\left(\Pi_{2} \mid t_{2}, b_{2}\right)$ over $b_{2} \in[6-\alpha, 6]$ can only be attained within $b_{2} \in[6-\alpha, 6-\alpha+\xi]$. 
Claim 2 (for the case $5-\alpha \leq b_{2} \leq 5$ ). There is an $M_{\xi}^{2} \in \mathbb{N}$ such that for every $k>M_{\xi}^{2}$ we have:

1. For every $t_{2} \in[\xi, 1]$, the maximum of $\mathbb{E}_{\beta_{1}^{k}}\left(\Pi_{2} \mid t_{2}, b_{2}\right)$ over $b_{2} \in[5-\alpha, 5]$ is attained at $b_{2}=5$.

2. For every $t_{2} \in[0, \xi)$, the maximum of $\mathbb{E}_{\beta_{1}^{k}}\left(\Pi_{2} \mid t_{2}, b_{2}\right)$ over $b_{2} \in[5-\alpha, 5]$ can only be attained within $b_{2} \in[5-\xi, 5]$.

Claim 3 (for case $5 \leq b_{2} \leq 6-\alpha$ ). There is an $M_{\xi}^{3} \in \mathbb{N}$ such that for every $k>M_{\xi}^{3}$ we have:

1. For every $t_{2} \in[\xi, 1-\xi]$, the maximum of $\mathbb{E}_{\beta_{1}^{k}}\left(\Pi_{2} \mid t_{2}, b_{2}\right)$ over $b_{2} \in[5,6-\alpha]$ can only be attained within $b_{2} \in\left[C_{M}\left(t_{2}\right)-\xi(1-\right.$ $\left.\alpha), C_{M}\left(t_{2}\right)+\xi(1-\alpha)\right]$.

2. For every $t_{2} \in[0, \xi)$, the maximum of $\mathbb{E}_{\beta_{1}^{k}}\left(\Pi_{2} \mid t_{2}, b_{2}\right)$ over $b_{2} \in[5,6-\alpha]$ can only be attained within $b_{2} \in[5,5+2 \xi(1-\alpha)]$.

3. For every $t_{2} \in(1-\xi, 1]$ the maximum of $\mathbb{E}_{\beta_{1}^{k}}\left(\Pi_{2} \mid t_{2}, b_{2}\right)$ over $b_{2} \in[5,6-\alpha]$ can only be attained within $b_{2} \in[6-\alpha-2 \xi(1-$ $\alpha), 6-\alpha]$.

It is important to note that part (1) of Claim 1 shows that for every $t_{2} \in[0,1-\xi]$, the maximum of $\mathbb{E}_{\beta_{1}^{k}}\left(\Pi_{2} \mid t_{2}, b_{2}\right)$ over $b_{2} \in[6-\alpha, 6]$ is not more than the maximum over $b_{2} \in[5,6-\alpha]$, which is verified in Claim 3. Similarly, part (1) of Claim 2 indicates that for every $t_{2} \in[\xi, 1]$, the maximum of $\mathbb{E}_{\beta_{1}^{k}}\left(\Pi_{2} \mid t_{2}, b_{2}\right)$ over $b_{2} \in[5-\alpha, 5]$ is not more than the maximum over $b_{2} \in[5,6-\alpha]$.

Proof of Claim 1. We compute $\mathbb{E}_{\beta_{1}^{k}}\left(\Pi_{2} \mid t_{2}, b_{2}\right)$ for every $b_{2} \in[6-\alpha, 6]$. Note that $C_{T}^{-1}\left(b_{2}\right) \in[0,1]$ is the unique type $t_{1}$ for bidder 1 such that $C_{T}\left(t_{1}\right)=b_{2}$. By the definition of $C_{T}$, we have $C_{T}^{-1}\left(b_{2}\right)=\sqrt{\frac{6-b_{2}}{\alpha}}$. Therefore, by (5)

$$
\begin{aligned}
\mathbb{E}_{\beta_{1}^{k}}\left(\Pi_{2} \mid t_{2}, b_{2}\right) & =\int_{0}^{1} \int_{5-\alpha}^{b_{2}}\left(5+t_{2}-\alpha t_{1}-b_{1}\right) \beta_{1}^{k}\left(t_{1}, d b_{1}\right) d t_{1} \\
& =\int_{0}^{C_{T}^{-1}\left(b_{2}\right)} \int_{5-\alpha}^{b_{2}}\left(5+t_{2}-\alpha t_{1}-b_{1}\right) \beta_{1}^{k}\left(t_{1}, d b_{1}\right) d t_{1} \\
& +\int_{C_{T}^{-1}\left(b_{2}\right)}^{1} \int_{5-\alpha}^{b_{2}}\left(5+t_{2}-\alpha t_{1}-b_{1}\right) \beta_{1}^{k}\left(t_{1}, d b_{1}\right) d t_{1} .
\end{aligned}
$$

From the definition of $\beta_{1}^{k}\left(t_{1}, \cdot\right)$ we obtain

$$
\begin{aligned}
& I=\left(1-2 \varepsilon_{k}-\varepsilon_{k}^{2}\right) \int_{0}^{C_{T}^{-1}\left(b_{2}\right)}\left(5+t_{2}-\alpha t_{1}-C_{M}\left(t_{1}\right)\right) d t_{1} \\
& +\varepsilon_{k} \int_{0}^{C_{T}^{-1}\left(b_{2}\right)}\left(5+t_{2}-\alpha t_{1}-C_{L}\left(t_{1}\right)\right) d t_{1} \\
& +\varepsilon_{k}^{2} \int_{0}^{C_{T}^{-1}\left(b_{2}\right)} \int_{5-\alpha}^{b_{2}}\left(5+t_{2}-\alpha t_{1}-b_{1}\right) \mu\left(d b_{1}\right) d t_{1} .
\end{aligned}
$$

Similarly, we have

$$
\begin{gathered}
\mathrm{II}=\left(1-2 \varepsilon_{k}-\varepsilon_{k}^{2}\right) \int_{C_{T}^{-1}\left(b_{2}\right)}^{1}\left(5+t_{2}-\alpha t_{1}-C_{M}\left(t_{1}\right)\right) d t_{1} \\
+\varepsilon_{k} \int_{C_{T}^{-1}\left(b_{2}\right)}^{1}\left(5+t_{2}-\alpha t_{1}-C_{L}\left(t_{1}\right)\right) d t_{1}
\end{gathered}
$$




$$
\begin{aligned}
& +\varepsilon_{k}^{2} \int_{C_{T}^{-1}\left(b_{2}\right)}^{1} \int_{5-\alpha}^{b_{2}}\left(5+t_{2}-\alpha t_{1}-b_{1}\right) \mu\left(d b_{1}\right) d t_{1} \\
& +\varepsilon_{k} \int_{C_{T}^{-1}\left(b_{2}\right)}^{1}\left(5+t_{2}-\alpha t_{1}-C_{T}\left(t_{1}\right)\right) d t_{1} .
\end{aligned}
$$

Hence, by the definition of the curves $C_{M}, C_{T}$, and $C_{L}$ we obtain

$$
\begin{aligned}
\mathbb{E}_{\beta_{1}^{k}}\left(\Pi_{2} \mid t_{2}, b_{2}\right)=\mathrm{I}+\mathrm{II} & =\left(1-2 \varepsilon_{k}-\varepsilon_{k}^{2}\right) \int_{0}^{1}\left(5+t_{2}-\alpha t_{1}-5-(1-\alpha) t_{1}\right) d t_{1} \\
& +\varepsilon_{k} \int_{0}^{1}\left(5+t_{2}-\alpha t_{1}-5+\alpha-\alpha\left(1-t_{1}\right)^{2}\right) d t_{1} \\
& +\varepsilon_{k}^{2} \int_{0}^{1} \int_{5-\alpha}^{b_{2}}\left(5+t_{2}-\alpha t_{1}-b_{1}\right) \mu\left(d b_{1}\right) d t_{1} \\
& +\varepsilon_{k} \int_{C_{T}^{-1}\left(b_{2}\right)}^{1}\left(5+t_{2}-\alpha t_{1}-6+\alpha t_{1}^{2}\right) d t_{1} .
\end{aligned}
$$

Thus,

$$
\begin{aligned}
\mathbb{E}_{\beta_{1}^{k}}\left(\Pi_{2} \mid t_{2}, b_{2}\right) & =\left(1-2 \varepsilon_{k}-\varepsilon_{k}^{2}\right)\left(t_{2}-\frac{1}{2}\right)+\varepsilon_{k}\left(t_{2}+\frac{\alpha}{6}\right) \\
& +\frac{\varepsilon_{k}^{2}}{1+\alpha} \int_{5-\alpha}^{b_{2}}\left(5+t_{2}-\frac{\alpha}{2}-b_{1}\right) d b_{1} \\
& +\varepsilon_{k} \int_{C_{T}^{-1}\left(b_{2}\right)}^{1}\left(-1+t_{2}-\alpha t_{1}+\alpha t_{1}^{2}\right) d t_{1} .
\end{aligned}
$$

Note that for every $t_{2} \in[0,1]$

$$
\begin{aligned}
\mathbb{E}_{\beta_{1}^{k}}\left(\Pi_{2} \mid t_{2}, 6-\alpha\right)-\mathbb{E}_{\beta_{1}^{k}}\left(\Pi_{2} \mid t_{2}, 6\right) & =-\frac{\varepsilon_{k}^{2}}{1+\alpha} \int_{6-\alpha}^{6}\left(5+t_{2}-\frac{\alpha}{2}-b_{1}\right) d b_{1} \\
& -\varepsilon_{k} \int_{0}^{1}\left(-1+t_{2}-\alpha t_{1}+\alpha t_{1}^{2}\right) d t_{1} \\
& =\left(\frac{\alpha}{1+\alpha}\right) \varepsilon_{k}^{2}\left(1-t_{2}\right)+\varepsilon_{k}\left(1-t_{2}+\frac{\alpha}{6}\right)>0,
\end{aligned}
$$

and also that $\mathbb{E}_{\beta_{1}^{k}}\left(\Pi_{2} \mid t_{2}, b_{2}\right)$ is continuous on the compact set $\left(t_{2}, b_{2}\right) \in[0,1] \times[5-\alpha, 6]$. Therefore, there is a $y>0$ (without loss of generality we can assume that $y \in(0, \alpha-\xi)$, in order to make the analysis easier) such that for every $t_{2} \in[0,1]$ and $b_{2} \in(6-y, 6]$

$$
\mathbb{E}_{\beta_{1}^{k}}\left(\Pi_{2} \mid t_{2}, 6-\alpha\right)>\mathbb{E}_{\beta_{1}^{k}}\left(\Pi_{2} \mid t_{2}, b_{2}\right) .
$$

This means that for every $t_{2} \in[0,1]$, bidding $6-\alpha$ is strictly better than any bid in the interval $(6-y, 6]$. Hence, it suffices to prove Claim 1 over $b_{2} \in[6-\alpha, 6-y]$. We do so by examining the first derivative of $\mathbb{E}_{\beta_{1}^{k}}\left(\Pi_{2} \mid t_{2}, b_{2}\right)$ with respect to $b_{2}$, which equals to 


$$
\frac{d}{d b_{2}} \mathbb{E}_{\beta_{1}^{k}}\left(\Pi_{2} \mid t_{2}, b_{2}\right)=\varepsilon_{k}^{2} \cdot q_{1}\left(t_{2}, b_{2}\right)+\varepsilon_{k} \cdot q_{2}\left(t_{2}, b_{2}\right),
$$

where

$$
\begin{aligned}
& q_{1}\left(t_{2}, b_{2}\right)=\frac{1}{1+\alpha} \cdot\left(5+t_{2}-\frac{\alpha}{2}-b_{2}\right) \\
& q_{2}\left(t_{2}, b_{2}\right)=\frac{1}{2 \alpha \cdot C_{T}^{-1}\left(b_{2}\right)} \cdot\left(-1+t_{2}-\alpha C_{T}^{-1}\left(b_{2}\right)+\alpha\left(C_{T}^{-1}\left(b_{2}\right)\right)^{2}\right) .
\end{aligned}
$$

Note that $q_{2}\left(t_{2}, b_{2}\right)$ is strictly negative for all $\left(t_{2}, b_{2}\right) \in[0,1] \times[6-\alpha, 6-y] \backslash\{(1,6-\alpha)\}$, and $q_{2}(1,6-\alpha)=0$.

Because $q_{2}$ is strictly negative on the compact set $[0,1-\xi] \times[6-\alpha, 6-y]$, it has a strictly negative upper-bound on $[0,1-\xi] \times[6-\alpha, 6-y]$. Therefore, since $\varepsilon_{k}^{2}$ is relatively much smaller than $\varepsilon_{k}$ for sufficiently large $k$, then $\frac{d}{d b_{2}} \mathbb{E}_{\beta_{1}^{k}}\left(\Pi_{2} \mid\right.$ $\left.t_{2}, b_{2}\right)<0$ for all $\left(t_{2}, b_{2}\right) \in[0,1-\xi] \times[6-\alpha, 6-y]$. This proves that there exists an $N_{\xi}^{1} \in \mathbb{N}$ such that for every $k>N_{\xi}^{1}$ we have $\frac{d}{d b_{2}} \mathbb{E}_{\beta_{1}^{k}}\left(\Pi_{2} \mid t_{2}, b_{2}\right)<0$ for all $\left(t_{2}, b_{2}\right) \in[0,1-\xi] \times[6-\alpha, 6-y]$. Hence, part (1) of Claim 1 holds for all $k>N_{\xi}^{1}$.

Because $q_{2}$ is strictly negative on the compact set $[1-\xi, 1] \times[6-\alpha+\xi, 6-y]$, we can apply a similar argument and find an $L_{\xi}^{1} \in \mathbb{N}$ such that part (2) of Claim 1 holds for every $k>L_{\xi}^{1}$. Therefore, by choosing $M_{\xi}^{1}=\max \left\{N_{\xi}^{1}, L_{\xi}^{1}\right\}$, Claim 1 is valid for every $k>M_{\xi}^{1}$.

Proof of Claim 2. We compute $\mathbb{E}_{\beta_{1}^{k}}\left(\Pi_{2} \mid t_{2}, b_{2}\right)$ for every $b_{2} \in[5-\alpha, 5]$. Note that type $C_{L}^{-1}\left(b_{2}\right) \in[0,1]$ is the unique type $t_{1}$ for bidder 1 such that $C_{L}\left(t_{1}\right)=b_{2}$. By the definition of $C_{L}$, we have $C_{L}^{-1}\left(b_{2}\right)=1-\sqrt{\frac{b_{2}-5}{\alpha}+1}$. Hence, by (5)

$$
\begin{aligned}
\mathbb{E}_{\beta_{1}^{k}}\left(\Pi_{2} \mid t_{2}, b_{2}\right) & =\int_{0}^{1} \int_{5-\alpha}^{b_{2}}\left(5+t_{2}-\alpha t_{1}-b_{1}\right) \beta_{1}^{k}\left(t_{1}, d b_{1}\right) d t_{1} \\
& =\int_{0}^{C_{L}^{-1}\left(b_{2}\right)} \int_{5-\alpha}^{b_{2}}\left(5+t_{2}-\alpha t_{1}-b_{1}\right) \beta_{1}^{k}\left(t_{1}, d b_{1}\right) d t_{1} \quad \text { III } \\
& +\int_{C_{L}^{-1}\left(b_{2}\right)}^{1} \int_{5-\alpha}^{b_{2}}\left(5+t_{2}-\alpha t_{1}-b_{1}\right) \beta_{1}^{k}\left(t_{1}, d b_{1}\right) d t_{1} . \quad \text { IV }
\end{aligned}
$$

Therefore, we have

$$
\begin{aligned}
& \mathrm{III}=\varepsilon_{k}^{2} \int_{0}^{C_{L}^{-1}\left(b_{2}\right)} \int_{5-\alpha}^{b_{2}}\left(5+t_{2}-\alpha t_{1}-b_{1}\right) \mu\left(d b_{1}\right) d t_{1}, \\
& \mathrm{IV}=\varepsilon_{k} \int_{C_{L}^{-1}\left(b_{2}\right)}^{1}\left(5+t_{2}-\alpha t_{1}-C_{L}\left(t_{1}\right)\right) d t_{1} \\
& +\varepsilon_{k}^{2} \int_{C_{L}^{-1}\left(b_{2}\right)}^{1} \int_{5-\alpha}^{b_{2}}\left(5+t_{2}-\alpha t_{1}-b_{1}\right) \mu\left(d b_{1}\right) d t_{1} .
\end{aligned}
$$

Hence, by the definition of the curve $C_{L}$ we obtain

$$
\begin{aligned}
\mathbb{E}_{\beta_{1}^{k}}\left(\Pi_{2} \mid t_{2}, b_{2}\right)=\mathrm{III}+\mathrm{IV} & =\varepsilon_{k}^{2} \int_{0}^{1} \int_{5-\alpha}^{b_{2}}\left(5+t_{2}-\alpha t_{1}-b_{1}\right) \mu\left(d b_{1}\right) d t_{1} \\
& +\varepsilon_{k} \int_{C_{L}^{-1}\left(b_{2}\right)}^{1}\left(5+t_{2}-\alpha t_{1}-5+\alpha-\alpha\left(1-t_{1}\right)^{2}\right) d t_{1}
\end{aligned}
$$




$$
\begin{aligned}
& =\frac{\varepsilon_{k}^{2}}{1+\alpha} \int_{5-\alpha}^{b_{2}}\left(5+t_{2}-\frac{\alpha}{2}-b_{1}\right) d b_{1} \\
& +\varepsilon_{k} \int_{C_{L}^{-1}\left(b_{2}\right)}^{1}\left(t_{2}+\alpha t_{1}-\alpha t_{1}^{2}\right) d t_{1} .
\end{aligned}
$$

Note that for every $t_{2} \in[0,1]$

$$
\begin{aligned}
\mathbb{E}_{\beta_{1}^{k}}\left(\Pi_{2} \mid t_{2}, 5\right)-\mathbb{E}_{\beta_{1}^{k}}\left(\Pi_{2} \mid t_{2}, 5-\alpha\right) & =\frac{\varepsilon_{k}^{2}}{1+\alpha} \int_{5-\alpha}^{5}\left(5+t_{2}-\frac{\alpha}{2}-b_{1}\right) d b_{1} \\
& +\varepsilon_{k} \int_{0}^{1}\left(t_{2}+\alpha t_{1}-\alpha t_{1}^{2}\right) d t_{1} \\
& =\left(\frac{\alpha}{1+\alpha}\right) \varepsilon_{k}^{2} t_{2}+\varepsilon_{k}\left(t_{2}+\frac{\alpha}{6}\right)>0,
\end{aligned}
$$

and also that $\mathbb{E}_{\beta_{1}^{k}}\left(\Pi_{2} \mid t_{2}, b_{2}\right)$ is continuous on the compact set $\left(t_{2}, b_{2}\right) \in[0,1] \times[5-\alpha, 5]$. Therefore, there is a $y>0$ (similar to the previous case assume that $y \in(0, \alpha-\xi))$ such that for every $t_{2} \in[0,1]$ and $b_{2} \in[5-\alpha, 5-\alpha+y$ )

$$
\mathbb{E}_{\beta_{1}^{k}}\left(\Pi_{2} \mid t_{2}, 5\right)>\mathbb{E}_{\beta_{1}^{k}}\left(\Pi_{2} \mid t_{2}, b_{2}\right) .
$$

This means that for every $t_{2} \in[0,1]$, bidding 5 is strictly better than any bid in the interval $[5-\alpha, 5-\alpha+y)$. Hence, it suffices to prove Claim 2 over the interval $[5-\alpha+y, 5]$. We do so by examining the first derivative of $\mathbb{E}_{\beta_{1}^{k}}\left(\Pi_{2} \mid t_{2}, b_{2}\right)$ with respect to $b_{2}$, which equals to

$$
\frac{d}{d b_{2}} \mathbb{E}_{\beta_{1}^{k}}\left(\Pi_{2} \mid t_{2}, b_{2}\right)=\varepsilon_{k}^{2} p_{1}\left(t_{2}, b_{2}\right)+\varepsilon_{k} p_{2}\left(t_{2}, b_{2}\right),
$$

where

$$
\begin{aligned}
& p_{1}\left(t_{2}, b_{2}\right)=\frac{1}{1+\alpha} \cdot\left(5+t_{2}-\frac{\alpha}{2}-b_{2}\right), \\
& p_{2}\left(t_{2}, b_{2}\right)=\frac{1}{2 \alpha \cdot\left(1-C_{L}^{-1}\left(b_{2}\right)\right)} \cdot\left(t_{2}+\alpha C_{L}^{-1}\left(b_{2}\right) 0-\alpha C_{L}^{-1}\left(b_{2}\right)^{2}\right) .
\end{aligned}
$$

Note that $p_{2}\left(t_{2}, b_{2}\right)$ is strictly positive for all $\left(t_{2}, b_{2}\right) \in[0,1] \times[5-\alpha+y, 5] \backslash\{(0,5)\}$, and $p_{2}(0,5)=0$. With an argument similar to that of the previous case, we can find an $M_{\xi}^{2}$ such that for every $k>M_{\xi}^{2}$ Claim 2 is valid.

Proof of Claim 3. We compute $\mathbb{E}_{\beta_{1}^{k}}\left(\Pi_{2} \mid t_{2}, b_{2}\right)$ for every $b_{2} \in[5,6-\alpha]$. Note that $C_{M}^{-1}\left(b_{2}\right)=\frac{b_{2}-5}{1-\alpha}$ for every $b_{2} \in[5,6-\alpha]$. With a similar argument as before we have

$$
\begin{aligned}
\mathbb{E}_{\beta_{1}^{k}}\left(\Pi_{2} \mid t_{2}, b_{2}\right) & =\left(1-2 \varepsilon_{k}-\varepsilon_{k}^{2}\right) \int_{0}^{\frac{b_{2}-5}{1-\alpha}}\left(5+t_{2}-\alpha t_{1}-C_{M}\left(t_{1}\right)\right) d t_{1} \\
& +\varepsilon_{k} \int_{0}^{1}\left(5+t_{2}-\alpha t_{1}-C_{L}\left(t_{1}\right)\right) d t_{1} \\
& +\varepsilon_{k}^{2} \int_{0}^{1} \int_{5-\alpha}^{b_{2}}\left(5+t_{2}-\alpha t_{1}-b_{1}\right) \mu\left(d b_{1}\right) d t_{1} .
\end{aligned}
$$

Hence, by the definition of the curves $C_{M}$ and $C_{L}$ we obtain 


$$
\begin{aligned}
\mathbb{E}_{\beta_{1}^{k}}\left(\Pi_{2} \mid t_{2}, b_{2}\right) & =\left(1-2 \varepsilon_{k}-\varepsilon_{k}^{2}\right) \int_{0}^{\frac{b_{2}-5}{1-\alpha}}\left(5+t_{2}-\alpha t_{1}-5-(1-\alpha) t_{1}\right) d t_{1} \\
& +\varepsilon_{k} \int_{0}^{1}\left(5+t_{2}-\alpha t_{1}-5+\alpha-\alpha\left(1-t_{1}\right)^{2}\right) d t_{1} \\
& +\varepsilon_{k}^{2} \int_{0}^{1} \int_{5-\alpha}^{b_{2}}\left(5+t_{2}-\alpha t_{1}-b_{1}\right) \mu\left(d b_{1}\right) d t_{1}
\end{aligned}
$$

Thus,

$$
\begin{aligned}
\mathbb{E}_{\beta_{1}^{k}}\left(\Pi_{2} \mid t_{2}, b_{2}\right) & =\left(1-2 \varepsilon_{k}-\varepsilon_{k}^{2}\right) \int_{0}^{\frac{b_{2}-5}{1-\alpha}}\left(t_{2}-t_{1}\right) d t_{1} \\
& +\varepsilon_{k} \int_{0}^{1}\left(t_{2}+\alpha t_{1}-\alpha t_{1}^{2}\right) d t_{1} \\
& +\frac{\varepsilon_{k}^{2}}{1+\alpha} \int_{5-\alpha}^{b_{2}}\left(5+t_{2}-\frac{\alpha}{2}-b_{1}\right) d b_{1} \\
& =\left(1-2 \varepsilon_{k}-\varepsilon_{k}^{2}\right)\left(t_{2}\left(\frac{b_{2}-5}{1-\alpha}\right)-\frac{1}{2}\left(\frac{b_{2}-5}{1-\alpha}\right)^{2}\right) \\
& +\varepsilon_{k}\left(t_{2}+\frac{\alpha}{6}\right) \\
& +\frac{\varepsilon_{k}^{2}}{2(1+\alpha)}\left(b_{2}-5+\alpha\right)\left(5+2 t_{2}-b_{2}\right) .
\end{aligned}
$$

So, the first derivative of $\mathbb{E}_{\beta_{1}^{k}}\left(\Pi_{2} \mid t_{2}, b_{2}\right)$ with respect to $b_{2}$ is

$$
\begin{aligned}
& \frac{d}{d b_{2}} \mathbb{E}_{\beta_{1}^{k}}\left(\Pi_{2} \mid t_{2}, b_{2}\right) \\
= & \frac{1-2 \varepsilon_{k}-\varepsilon_{k}^{2}}{1-\alpha} \cdot\left(t_{2}-\frac{b_{2}-5}{1-\alpha}\right)+\frac{\varepsilon_{k}^{2}}{1+\alpha}\left(5+t_{2}-\frac{\alpha}{2}-b_{2}\right) .
\end{aligned}
$$

First, we prove that for every $t_{2} \in[\xi, 1-\xi]$ the maximum of $\mathbb{E}_{\beta_{1}^{k}}\left(\Pi_{2} \mid t_{2}, b_{2}\right)$ cannot be attained on the boundary of interval $[5,6-\alpha]$. Observe that

$$
\frac{d}{d b_{2}} \mathbb{E}_{\beta_{1}^{k}}\left(\Pi_{2} \mid t_{2}, 5\right)=\frac{1-2 \varepsilon_{k}-\varepsilon_{k}^{2}}{1-\alpha} \cdot t_{2}+\frac{\varepsilon_{k}^{2}}{1+\alpha}\left(t_{2}-\frac{\alpha}{2}\right),
$$

and

$$
\frac{d}{d b_{2}} \mathbb{E}_{\beta_{1}^{k}}\left(\Pi_{2} \mid t_{2}, 6-\alpha\right)=\frac{1-2 \varepsilon_{k}-\varepsilon_{k}^{2}}{1-\alpha} \cdot\left(t_{2}-1\right)+\frac{\varepsilon_{k}^{2}}{1+\alpha}\left(t_{2}-1+\frac{\alpha}{2}\right) .
$$

Hence, there is an $N_{\xi}^{3} \in \mathbb{N}$ such that if $k>N_{\xi}^{3}$ then for every $t_{2} \in[\xi, 1-\xi]$ we have

$$
\frac{d}{d b_{2}} \mathbb{E}_{\beta_{1}^{k}}\left(\Pi_{2} \mid t_{2}, 5\right)>0
$$

and

$$
\frac{d}{d b_{2}} \mathbb{E}_{\beta_{1}^{k}}\left(\Pi_{2} \mid t_{2}, 6-\alpha\right)<0 .
$$

Therefore, by the continuity of $\mathbb{E}_{\beta_{1}^{k}}\left(\Pi_{2} \mid t_{2}, b_{2}\right)$, we can conclude for every $t_{2} \in[\xi, 1-\xi]$ that if $b_{2}$ maximizes $\mathbb{E}_{\beta_{1}^{k}}\left(\Pi_{2} \mid\right.$ $\left.t_{2}, b_{2}\right)$, then 


$$
\frac{d}{d b_{2}} \mathbb{E}_{\beta_{1}^{k}}\left(\Pi_{2} \mid t_{2}, b_{2}\right)=0
$$

One can check with the help of (7) that the unique solution of (8), for every $t_{2} \in[0,1]$, is

$$
b_{2}\left(t_{2}\right)=\frac{\left(1-2 \varepsilon_{k}-\varepsilon_{k}^{2}\right)(1+\alpha)}{v\left(\varepsilon_{k}\right)} \cdot\left(5+(1-\alpha) t_{2}\right)+\frac{\varepsilon_{k}^{2}(1-\alpha)^{2}}{v\left(\varepsilon_{k}\right)}\left(5+t_{2}-\frac{\alpha}{2}\right),
$$

where

$$
v\left(\varepsilon_{k}\right)=\left(1-2 \varepsilon_{k}-\varepsilon_{k}^{2}\right)(1+\alpha)+\varepsilon_{k}^{2}(1-\alpha)^{2} .
$$

One can verify that

$$
\left|b_{2}\left(t_{2}\right)-C_{M}\left(t_{2}\right)\right|=\frac{\alpha \varepsilon_{k}^{2}(1-\alpha)^{2}}{v\left(\varepsilon_{k}\right)}\left|t_{2}-\frac{1}{2}\right| \leq \frac{\alpha \varepsilon_{k}^{2}(1-\alpha)^{2}}{2 v\left(\varepsilon_{k}\right)} .
$$

Therefore, there is an $L_{\xi}^{3} \in \mathbb{N}$ such that for every $k>L_{\xi}^{3}$ and every $t_{2} \in[0,1]$ we have

$$
\left|b_{2}\left(t_{2}\right)-C_{M}\left(t_{2}\right)\right| \leq \xi(1-\alpha) .
$$

By choosing $M_{\xi}^{3}=\max \left\{N_{\xi}^{3}, L_{\xi}^{3}\right\}$, for every $k>M_{\xi}^{3}$, part (1) of Claim 3 is valid.

Since at $t_{2}=0$, for every $b_{2} \in[5,6-\alpha]$ we have

$$
\frac{d}{d b_{2}} \mathbb{E}_{\beta_{1}^{k}}\left(\Pi_{2} \mid 0, b_{2}\right)=\frac{1-2 \varepsilon_{k}-\varepsilon_{k}^{2}}{1-\alpha} \cdot\left(-\frac{b_{2}-5}{1-\alpha}\right)+\frac{\varepsilon_{k}^{2}}{1+\alpha}\left(5-\frac{\alpha}{2}-b_{2}\right)<0,
$$

the maximum at $t_{2}=0$ is obtained at $b_{2}=5$. Moreover, by part (1) of Claim 3 we have that if $k>\max \left\{N_{\xi}^{3}, L_{\xi}^{3}\right\}$, then for $t_{2}=\xi$ the maximum is obtained within $b_{2} \in[5,5+2(1-\alpha) \xi]$. One can verify that in (7), the first derivative of unique solution $b_{2}\left(t_{2}\right)$ is strictly positive, then we can conclude that for every $t_{2} \in[0, \xi)$ the maximum is obtained within $b_{2} \in$ $[5,5+2(1-\alpha) \xi]$, for large $k$.

With a similar argument, since at $t_{2}=1$ for every $b_{2} \in[5,6-\alpha]$ we have

$$
\frac{d}{d b_{2}} \mathbb{E}_{\beta_{1}^{k}}\left(\Pi_{2} \mid 1, b_{2}\right)=\frac{1-2 \varepsilon_{k}-\varepsilon_{k}^{2}}{1-\alpha} \cdot\left(1-\frac{b_{2}-5}{1-\alpha}\right)+\frac{\varepsilon_{k}^{2}}{1+\alpha}\left(6-\frac{\alpha}{2}-b_{2}\right)>0,
$$

the maximum at $t_{2}=1$ is obtained in $b_{2}=6-\alpha$. Moreover, if $k>\max \left\{N_{\xi}^{3}, L_{\xi}^{3}\right\}$, then for $t_{2}=1-\xi$ the maximum is obtained within $b_{2} \in[6-\alpha-2(1-\alpha) \xi, 6-\alpha]$. Similarly, since $b_{2}{ }^{\prime}\left(t_{2}\right)$ is strictly positive, we conclude that for every $t_{2} \in(1-\xi, 1]$ the maximum is obtained within $b_{2} \in[6-\alpha-2(1-\alpha) \xi, 6-\alpha]$ for large $k$. This complete the proof of Claim 3 , for every $k>M_{\xi}^{3}$.

\subsection{Proofs of Propositions 27 and 28}

It is easy to check that strategy profiles $\sigma$ and $\eta$ are BNEs.

We now prove that the BNE $\sigma=\left(\sigma_{1}, \sigma_{2}\right)$, in which $\sigma_{1}\left(t_{1}, \cdot\right)=\delta_{6}(\cdot)$ and $\sigma_{2}\left(t_{2}, \cdot\right)=\delta_{5-\alpha}(\cdot)$, is not pointwise-perfect, consequently is not uniform-perfect. Let $\left(\sigma_{1}^{k}\right)_{k=1}^{\infty}$ be a sequence of completely mixed strategies for bidder 1 such that $\rho^{w}\left(\sigma_{1}^{k}, \sigma_{1}\right) \longrightarrow 0$ as $k \rightarrow \infty$. We show that bidder's 2 best response against $\sigma_{1}^{k}$ does not converge to $\sigma_{2}$ when $k \rightarrow \infty$.

Suppose that bidder 1 plays $\sigma_{1}^{k}$, for some $k \in \mathbb{N}$. We prove that for $t_{2}=1$, the best response of bidder 2 is far from $\sigma_{2}\left(t_{2}, \cdot\right)=\delta_{5-\alpha}(\cdot)$. Note that bidder 2 gets always zero by choosing $\sigma_{2}$, because he never wins. So, it is enough to show that his expected profit is strictly positive by bidding $6-\alpha$, when $t_{2}=1$. We compute bidder 2 's expected profit given type $t_{2}=1$ and bid $b_{2}=6-\alpha$.

$$
\mathbb{E}_{\sigma_{1}^{k}}\left(\Pi_{2} \mid 1,6-\alpha\right)=\int_{0}^{1} \int_{5-\alpha}^{6-\alpha}\left(5+1-\alpha t_{1}-b_{1}\right) \sigma_{1}^{k}\left(t_{1}, d b_{1}\right) d t_{1} .
$$

Since $\sigma_{1}^{k}$ is a completely mixed strategy we have $\sigma_{1}^{k}\left(t_{1},(5-\alpha, 6-\alpha)\right)>0$, therefore by using the fact that $b_{1} \in(5-\alpha$, $6-\alpha$ ) we have

$$
\begin{aligned}
\mathbb{E}_{\sigma_{1}^{k}}\left(\Pi_{2} \mid 1,6-\alpha\right)> & \int_{0}^{1} \int_{5-\alpha}^{6-\alpha}\left(5+1-\alpha t_{1}-6+\alpha\right) \sigma_{1}^{k}\left(t_{1}, d b_{1}\right) d t_{1} \\
& =\alpha \int_{0}^{1} \int_{5-\alpha}^{6-\alpha}\left(1-t_{1}\right) \sigma_{1}^{k}\left(t_{1}, d b_{1}\right) d t_{1} \geq 0 .
\end{aligned}
$$

This implies that $\mathbb{E}_{\sigma_{1}^{k}}\left(\Pi_{2} \mid 1,6-\alpha\right)>0$, hence $\sigma_{2}$ is not pointwise-perfect. 
With a similar argument, one can prove that the discontinuous BNE $\eta$ is not pointwise-perfect, and hence not uniformperfect either.

\section{Appendix III: A measurability result for the weak distance}

A strategy $\beta_{i}$ for some player $i$ is said to be countably supported if there exists a countable set $B \subseteq A_{i}$ such that $\beta_{i}\left(t_{i}, B\right)=1$ for every $t_{i} \in T_{i}$.

Lemma 31. Assume that the action space $A_{i}$ is separable for a player $i \in N$. Then, for every strategy $\beta_{i}$ for player $i$, there exists a sequence $\left(\tau_{i}^{k}\right)_{k=1}^{\infty}$ of countably supported strategies such that

$$
\lim _{k \rightarrow \infty} \rho^{w}\left(\tau_{i}^{k}\left(t_{i}, \cdot\right), \beta_{i}\left(t_{i}, \cdot\right)\right)=0
$$

for every type $t_{i} \in T_{i}$.

Proof. Part 1: The definition of the sequence $\left(\tau_{i}^{k}\right)_{k=1}^{\infty}$ : Since $A_{i}$ is separable, it has a countable dense subset $A_{i}^{*}=\left\{a_{j}\right\}_{j \in \mathbb{N}}$.

Take a $k \in \mathbb{N}$. For every $a \in A_{i}$, let $U^{k}(a)=\left\{a^{\prime} \in A_{i} \mid d_{A_{i}}\left(a, a^{\prime}\right)<\frac{1}{k}\right\}$. Let $B_{1}^{k}=U^{k}\left(a_{1}\right)$, and then define recursively $B_{j}^{k}=$ $U^{k}\left(a_{j}\right) \backslash \cup_{\ell=1}^{j-1} B_{\ell}$ for every $j \in \mathbb{N}$ with $j \geq 2$. Let

$$
J^{k}=\left\{j \in \mathbb{N} \mid B_{j}^{k} \neq \emptyset\right\} .
$$

Since $A_{i}^{*}$ is dense in $A_{i}$, it follows that $\left\{B_{j}^{k} \mid j \in J^{k}\right\}$ is a partition of $A_{i}$. Moreover, by construction, $B_{j}^{k} \in \mathcal{A}_{i}$ for every $j \in J^{k}$. Take an arbitrary $b_{j}^{k} \in B_{j}^{k}$ for every $j \in J^{k}$.

Now define the countably supported strategy $\tau_{i}^{k}$ for player $i$ by

$$
\tau_{i}^{k}\left(t_{i}, \cdot\right)=\sum_{j \in J^{k}} \beta_{i}\left(t_{i}, B_{j}^{k}\right) \cdot \delta_{b_{j}^{k}}(\cdot)
$$

for every $t_{i} \in T_{i}$, where as usual, $\delta$ stands for the Dirac measure. Note that $\tau_{i}^{k}$ satisfies condition (2) of the definition of behavior strategies, because for every $Z \in \mathcal{A}_{i}$

$$
\tau_{i}^{k}(\cdot, Z)=\sum_{j \in J^{k}: b_{j}^{k} \in Z} \beta_{i}\left(\cdot, B_{j}^{k}\right) .
$$

Part 2: The proof that the sequence $\left(\tau_{i}^{k}\right)_{k=1}^{\infty}$ satisfies equality (9): Fix a type $t_{i} \in T_{i}$. Consider a bounded Lipschitz function $f: A_{i} \rightarrow \mathbb{R}$. Then, there exists $C \geq 0$ such that $\left|f(a)-f\left(a^{\prime}\right)\right| \leq C \cdot d_{A_{i}}\left(a, a^{\prime}\right)$ for every $a, a^{\prime} \in A_{i}$. In particular, for every $j \in J^{k}$, since the diameter of $B_{j}^{k}$ is at most $\frac{2}{k}$, we have $\left|f(a)-f\left(a^{\prime}\right)\right| \leq C \cdot \frac{2}{k}$ for every $a, a^{\prime} \in B_{j}^{k}$. Thus,

$$
\begin{aligned}
\left|\int_{A_{i}} f(a) \tau_{i}^{k}\left(t_{i}, d a\right)-\int_{A_{i}} f(a) \beta_{i}\left(t_{i}, d a\right)\right| & \leq \sum_{j \in J^{k}}\left|\int_{B_{j}^{k}} f(a) \tau_{i}^{k}\left(t_{i}, d a\right)-\int_{B_{j}^{k}} f(a) \beta_{i}\left(t_{i}, d a\right)\right| \\
& =\sum_{j \in J^{k}}\left|f\left(b_{j}^{k}\right) \cdot \beta_{i}\left(t_{i}, B_{j}^{k}\right)-\int_{B_{j}^{k}} f(a) \beta_{i}\left(t_{i}, d a\right)\right| \\
& =\sum_{j \in J^{k}}\left|\int_{B_{j}^{k}}\left[f\left(b_{j}^{k}\right)-f(a)\right] \beta_{i}\left(t_{i}, d a\right)\right| \\
& \leq \sum_{j \in J^{k}} \sup _{a \in B_{j}^{k}}\left|f\left(b_{j}^{k}\right)-f(a)\right| \cdot \beta_{i}\left(t_{i}, B_{j}^{k}\right) \\
& \leq \sum_{j \in J^{k}} C \cdot \frac{2}{k} \cdot \beta_{i}\left(t_{i}, B_{j}^{k}\right) \\
& =C \cdot \frac{2}{k} \cdot
\end{aligned}
$$


Hence, $\int_{A_{i}} f(a) \tau_{i}^{k}\left(t_{i}, d a\right)$ converges to $\int_{A_{i}} f(a) \beta_{i}\left(t_{i}, d a\right)$ as $k$ tends to infinity. Because $f$ was an arbitrary bounded Lipschitz function from $A_{i}$ to $\mathbb{R}$, the proof of (9) is complete.

Lemma 32. Assume that the action space $A_{i}$ is separable for a player $i \in N$, and let $\beta_{i}^{1}$ and $\beta_{i}^{2}$ be two strategies for player $i$. Then, the function $f: T_{i} \rightarrow \mathbb{R}$ defined by $f\left(t_{i}\right)=\rho^{w}\left(\beta_{i}^{1}\left(t_{i}, \cdot\right), \beta_{i}^{2}\left(t_{i}, \cdot\right)\right)$ is measurable.

Proof. It suffices to prove that the set $\left\{t_{i} \in T_{i} \mid f\left(t_{i}\right)>r\right\}$ is measurable for every $r \in \mathbb{R}$. So, fix an arbitrary $r \in \mathbb{R}$.

Part 1: when $\beta_{i}^{1}$ and $\beta_{i}^{2}$ are countably supported strategies.

First assume that $\beta_{i}^{1}$ and $\beta_{i}^{2}$ are countably supported strategies. Then, there are countable sets $B^{1}, B^{2} \subseteq A_{i}$ such that $\beta_{i}^{1}\left(t_{i}, B^{1}\right)=1$ and $\beta_{i}^{2}\left(t_{i}, B^{2}\right)=1$ for every $t_{i} \in T_{i}$. Let $B=B^{1} \cup B^{2}$. We have

$$
f\left(t_{i}\right)=\inf \left\{\varepsilon>0 \mid \beta_{i}^{1}\left(t_{i}, C\right) \leq \beta_{i}^{2}\left(t_{i}, C^{\varepsilon}\right)+\varepsilon \text { and } \beta_{i}^{2}\left(t_{i}, C\right) \leq \beta_{i}^{1}\left(t_{i}, C^{\varepsilon}\right)+\varepsilon \forall C \in \mathcal{A}_{i}\right\} .
$$

Notice that if $\beta_{i}^{1}\left(t_{i}, C\right) \leq \beta_{i}^{2}\left(t_{i}, C^{\varepsilon}\right)+\varepsilon$ for some $C \subseteq B$, then for any $\widetilde{C} \in \mathcal{A}_{i}$ satisfying $\widetilde{C} \cap B=C$, we obtain

$$
\beta_{i}^{1}\left(t_{i}, \widetilde{C}\right)=\beta_{i}^{1}\left(t_{i}, C\right) \leq \beta_{i}^{2}\left(t_{i}, C^{\varepsilon}\right)+\varepsilon \leq \beta_{i}^{2}\left(t_{i}, \widetilde{C}^{\varepsilon}\right)+\varepsilon .
$$

Similarly, if $\beta_{i}^{2}\left(t_{i}, C\right) \leq \beta_{i}^{1}\left(t_{i}, C^{\varepsilon}\right)+\varepsilon$ then

$$
\beta_{i}^{2}\left(t_{i}, \widetilde{C}\right) \leq \beta_{i}^{1}\left(t_{i}, \widetilde{C}^{\varepsilon}\right)+\varepsilon
$$

Hence,

$$
f\left(t_{i}\right)=\inf \left\{\varepsilon>0 \mid \beta_{i}^{1}\left(t_{i}, C\right) \leq \beta_{i}^{2}\left(t_{i}, C^{\varepsilon}\right)+\varepsilon \text { and } \beta_{i}^{2}\left(t_{i}, C\right) \leq \beta_{i}^{1}\left(t_{i}, C^{\varepsilon}\right)+\varepsilon \forall C \subseteq B\right\} .
$$

Notice that $f\left(t_{i}\right)>r$ holds if and only if there exist an $m \in \mathbb{N}$ and a set $C \subseteq B$ such that for $\varepsilon=r+\frac{1}{m}$ we have either $\beta_{i}^{1}\left(t_{i}, C\right)>\beta_{i}^{2}\left(t_{i}, C^{\varepsilon}\right)+\varepsilon$ or $\beta_{i}^{2}\left(t_{i}, C\right)>\beta_{i}^{1}\left(t_{i}, C^{\varepsilon}\right)+\varepsilon$. Indeed, the "only if"-part is immediate, whereas the "if"-part follows from the fact that $\beta_{i}^{2}\left(t_{i}, C^{\varepsilon}\right)+\varepsilon$ and $\beta_{i}^{1}\left(t_{i}, C^{\varepsilon}\right)+\varepsilon$ are increasing in $\varepsilon$.

As $B$ is countable, we can even restrict to finite subsets of $B$ : thus $f\left(t_{i}\right)>r$ holds if and only if there exist an $m \in \mathbb{N}$ and a finite set $C \subseteq B$ such that for $\varepsilon=r+\frac{1}{m}$ we have either $\beta_{i}^{1}\left(t_{i}, C\right)>\beta_{i}^{2}\left(t_{i}, C^{\varepsilon}\right)+\varepsilon$ or $\beta_{i}^{2}\left(t_{i}, C\right)>\beta_{i}^{1}\left(t_{i}, C^{\varepsilon}\right)+\varepsilon$.

For every $m \in \mathbb{N}$ and finite $C \subseteq B$, define

$$
\begin{aligned}
& U_{m, C}^{1}=\left\{t_{i} \in T_{i} \mid \beta_{i}^{1}\left(t_{i}, C\right)>\beta_{i}^{2}\left(t_{i}, C^{r+\frac{1}{m}}\right)+r+\frac{1}{m}\right\} \\
& U_{m, C}^{2}=\left\{t_{i} \in T_{i} \mid \beta_{i}^{2}\left(t_{i}, C\right)>\beta_{i}^{1}\left(t_{i}, C^{r+\frac{1}{m}}\right)+r+\frac{1}{m}\right\} .
\end{aligned}
$$

Since by condition (2) of the definition of behavior strategies, the section functions $\beta_{i}^{1}(\cdot, C): T_{i} \rightarrow \mathbb{R}$ and $\beta_{i}^{2}\left(\cdot, C^{r+\frac{1}{m}}\right): T_{i} \rightarrow$ $\mathbb{R}$ are measurable, the sets $U_{m, C}^{1}$ are measurable. For a similar reason, the sets $U_{m, C}^{2}$ are measurable too. Now we have

$$
\left\{t_{i} \in T_{i} \mid f\left(t_{i}\right)>r\right\}=\bigcup_{m=1}^{\infty} \bigcup_{\text {finite } C \subseteq B}\left[U_{m, C}^{1} \bigcup U_{m, C}^{2}\right] .
$$

Because the right hand side is a countable union of measurable sets, the set $\left\{t_{i} \in T_{i} \mid f\left(t_{i}\right)>r\right\}$ is measurable as well. So, the claim of the lemma holds for countably supported strategies.

\section{Part 2: when $\beta_{i}^{1}$ and $\beta_{i}^{2}$ are arbitrary strategies.}

Now we prove the lemma for arbitrary strategies $\beta_{i}^{1}$ and $\beta_{i}^{2}$. By Lemma 31, there are two sequences of countably supported strategies $\left(\tau_{i}^{k, 1}\right)_{k=1}^{\infty}$ and $\left(\tau_{i}^{k, 2}\right)_{k=1}^{\infty}$ such that

$$
\lim _{k \rightarrow \infty} \rho^{w}\left(\tau_{i}^{k, 1}\left(t_{i}, \cdot\right), \beta_{i}^{1}\left(t_{i}, \cdot\right)\right)=0, \quad \text { and } \quad \lim _{k \rightarrow \infty} \rho^{w}\left(\tau_{i}^{k, 2}\left(t_{i}, \cdot\right), \beta_{i}^{2}\left(t_{i}, \cdot\right)\right)=0
$$

for every type $t_{i} \in T_{i}$. By the definition of $f$, we have

$$
\left\{t_{i} \in T_{i} \mid f\left(t_{i}\right)>r\right\}=\left\{t_{i} \in T_{i} \mid \lim _{k \rightarrow \infty} \rho^{w}\left(\tau_{i}^{k, 1}\left(t_{i}, \cdot\right), \tau_{i}^{k, 2}\left(t_{i}, \cdot\right)\right)>r\right\} .
$$

Notice that, for some $t_{i} \in T_{i}$, the inequality

$$
\lim _{k \rightarrow \infty} \rho^{w}\left(\tau_{i}^{k, 1}\left(t_{i}, \cdot\right), \tau_{i}^{k, 2}\left(t_{i}, \cdot\right)\right)>r
$$


holds if and only if there exists an $m \in \mathbb{N}$ such that $\rho^{w}\left(\tau_{i}^{k, 1}\left(t_{i}, \cdot\right), \tau_{i}^{k, 2}\left(t_{i}, \cdot\right)\right)>r+\frac{1}{m}$ holds for sufficiently large $k$. Therefore,

$$
\left\{t_{i} \in T_{i} \mid f\left(t_{i}\right)>r\right\}=\bigcup_{m=1}^{\infty} \bigcup_{\ell=1}^{\infty} \bigcap_{k=\ell}^{\infty}\left\{t_{i} \in T_{i} \mid \rho^{w}\left(\tau_{i}^{k, 1}\left(t_{i}, \cdot\right), \tau_{i}^{k, 2}\left(t_{i}, \cdot\right)\right)>r+\frac{1}{m}\right\} .
$$

For each $k \in \mathbb{N}$, because the strategies $\tau_{i}^{k, 1}$ and $\tau_{i}^{k, 2}$ are countably supported, part 1 of the proof implies that set

$$
\left\{t_{i} \in T_{i} \mid \rho^{w}\left(\tau_{i}^{k, 1}\left(t_{i}, \cdot\right), \tau_{i}^{k, 2}\left(t_{i}, \cdot\right)\right)>r+\frac{1}{m}\right\}
$$

is measurable. Therefore, the set $\left\{t_{i} \in T_{i} \mid f\left(t_{i}\right)>r\right\}$ is measurable as well.

\section{Acknowledgments}

The authors would like to thank the associate editor and two anonymous referees for their very helpful suggestions and remarks.

\section{References}

Aliprantis, C.D., Border, C., 1999. Infinite Dimensional Analysis. Springer-Verlag.

Aumann, R.J., 1964. Mixed and behavior strategies in infinite extensive games. In: Advances in Game Theory. In: Annals of Mathematical Studies, vol. 52. Princeton University Press, Princeton, NJ, pp. 627-650.

Bajoori, E., Flesch, J., Vermeulen, D., 2013. Perfect equilibrium in games with compact action spaces. Games Econ. Behav. 82, $490-502$.

Bajoori, E., 2011. Distributional perfect equilibrium in Bayesian games. Working paper.

Balder, E., 1988. Generalized equilibrium results for games with incomplete information. Math. Oper. Res. 13 (2), $265-276$.

Balder, E., 2002. A unifying pair of Cournot-Nash equilibrium existence results. J. Econ. Theory 102, 437-470.

Billingsley, P., 1968. Convergence of Probability Measures. Wiley Series in Probability and Mathematical Statistics. John Wiley \& Sons.

Bonanno, G., 2013. AGM-consistency and perfect Bayesian equilibrium. Part I: definition and properties. Int. J. Game Theory 42, 567-592.

Dudley, R.M., 2002. Real Analysis and Probability. Cambridge University Press.

Fudenberg, D., Tirole, J., 1991. Perfect Bayesian equilibrium and sequential equilibrium. J. Econ. Theory 53, 236-260.

Govindan, S., 1995. Stability and the chain store paradox. J. Econ. Theory 66, 536-547.

Hellman, Z., Levy, Y., 2013. Bayesian games with a continuum of states. Working paper.

Jackson, M.O., Simon, L.K., Swinkels, J.M., Zame, W.R., 2002. Communication and equilibrium in discontinuous games of incomplete information. Econometrica $70,1711-1740$.

Jackson, M.O., Simon, L.K., Swinkels, J.M., Zame, W.R., 2004. Corrigendum to "Communication and equilibrium in discontinuous games of incomplete information". Econometrica 72, 1927-1929.

Kohlberg, E., Mertens, J-F., 1986. On the strategic stability of equilibria. Econometrica 54, 1003-1037.

Kreps, D.M., Wilson, R., 1982. Sequential equilibria. Econometrica 50, 863-894.

Laffont, J.J., Martimort, D., 2001. The Theory of Incentives: The Principal-Agent Model. Princeton University Press.

Liu, H., 2014. Equilibrium selection in common-value second-price auctions. Games Econ. Behav. 84, 1-6.

Mertens, J-F., 1989. Stable equilibria - a reformulation, part I. Math. Oper. Res. 14, 575-624.

Mertens, J-F., 1991. Stable equilibria - a reformulation, part II: discussion of the definition and further results. Math. Oper. Res. 16 , 694-753.

Mertens, J.F., 2003. Ordinality in non cooperative games. Int. J. Game Theory 32, 387-430.

Milgrom, P.R., Weber, R.J., 1985. Distributional strategies for games with incomplete information. Math. Oper. Res. 10, 619-632.

Myerson, R.B., Reny, P.J., 2011. Sequential equilibria of multi-stage games with infinite sets of types and actions. Working paper.

Nash, J.F., 1951. Non-cooperative games. Ann. Math. 54 (2), 286-295.

Parthasarathy, K.R., 1967. Probability Measures on Metric Spaces. Academic Press, New York, London.

Prokhorov, Y.V., 1956. Convergence of random processes and limit theorems in probability theory. Theory Probab. Appl. 1, 157-214.

Reny, P.J., 1999. On the existence of pure and mixed strategy Nash equilibria in discontinuous games. Econometrica 67, 1029-1056.

Reny, P.J., 2011. On the existence of monotone pure strategy equilibrium in Bayesian games. Econometrica 79, 499-553.

Selten, R., 1975. Reexamination of the perfectness concept for equilibrium points in extensive games. Int. J. Game Theory 4 , $25-55$.

Selten, R., 1978. The chain store paradox. Theory Dec. 9, 127-159.

Simon, R.S., 2003. Games of incomplete information, ergodic theory, and the measurability of equilibria. Isr. J. Math. 138, 73-92.

Simon, L.K., Stinchcombe, M.M., 1995. Equilibrium refinement for infinite normal-form games. Econometrica 63, 1421-1443.

Vermeulen, A.J., Jansen, M.J.M., 1997. On the invariance of solutions of finite games. Math. Soc. Sci. 33, $251-267$. 